\title{
F-Term Hybrid Inflation Followed by a Peccei-Quinn Phase Transition
}

\author{
G. Lazarides ${ }^{1, *}$ and C. Pallis ${ }^{2, \dagger}$ \\ ${ }^{1}$ Physics Division, School of Technology, Aristotle University of Thessaloniki, Thessaloniki 54124, GREECE \\ ${ }^{2}$ Department of Physics, University of Cyprus, P.O. Box 20537, Nicosia 1678, CYPRUS
}

\begin{abstract}
We consider a cosmological set-up, based on renormalizable superpotential terms, in which a superheavy scale F-term hybrid inflation is followed by a Peccei-Quinn phase transition, resolving the strong CP and $\mu$ problems of the minimal supersymmetric standard model. We show that the field which triggers the Peccei-Quinn phase transition can remain after inflation well above the Peccei-Quinn scale thanks to (i) its participation in the supergravity and logarithmic corrections during the inflationary stage and (ii) the high reheat temperature after the same period. As a consequence, its presence influences drastically the inflationary dynamics and the universe suffers a second period of reheating after the Peccei-Quinn phase transition. Confronting our inflationary predictions with the current observational data, we find that, for about the central value of the spectral index, the grand unification scale can be identified with its supersymmetric value for the relevant coupling constant $\kappa \simeq 0.002$ and, more or less, natural values, $\pm(0.01-0.1)$, for the remaining parameters. On the other hand, the final reheat temeperature after the Peccei-Quinn phase transition turns out to be low enough so as the gravitino problem is avoided.
\end{abstract}

PACS numbers: $98.80 . \mathrm{Cq}$

\section{INTRODUCTION}

One of the most natural and well-motivated inflationary model is the supersymmetric (SUSY) F-term hybrid inflation (FHI) [1, 2]. It is realized at (or close to) the SUSY Grand Unified Theory (GUT) scale $M_{\mathrm{GUT}} \simeq$ $2.86 \times 10^{16} \mathrm{GeV}$ and can be easily linked to extensions [3] of the Minimal Supersymmetric Standard Model (MSSM) which provide solutions to a number of problems of MSSM. Namely, the $\mu$-problem of MSSM can be solved via a direct coupling of the inflaton to Higgs superfields [4] or via a Peccei-Quinn (PQ) symmetry [5] which also solves the strong CP problem [6]. Also baryon number conservation can be an automatic consequence [4] of a $\mathrm{R}$ symmetry and the baryon asymmetry of the universe can be generated via leptogenesis which takes place 7] through the out-of-equilibrium decay of the inflaton's decay products.

The aforementioned resolution of the $\mu$-problem of MSSM via a PQ symmetry can be achieved [5] by considering non-renormalizable superpotential terms involving additional singlets which develop vacuum expectation values (VEVs) of the order of the PQ symmetry breaking scale. The emerging potential of these singlets has a local minimum which is separated from the global PQ minimum by a sizable potential barrier preventing a successful transition from the trivial to the $\mathrm{PQ}$ vacuum. As one can show [8], by considering the one-loop temperature corrections [9] to the scalar potential, this situation persists at all cosmic temperatures after reheating. It is, thus, obligatory to assume that, after the termination of FHI, the system emerges with the appropriate combina-

\footnotetext{
*Electronic address: lazaride@eng.auth.gr
}

${ }^{\dagger}$ Electronic address: kpallis@gen.auth.gr tion of initial conditions so that it is led [10] to the PQ vacuum. Stated differently, the PQ symmetry is to be broken during or before FHI. As a consequence, tight upper bounds on the inflationary scale have to be imposed so that the isocurvature fluctuations [11] of the axion are consistent with the observational bounds - this restriction, though, can be alleviated when cosmic strings are formed [12].

This latter complication can be avoided, if the $P Q$ phase transition (PQPT) takes place after the end of FHI. This possibility can be realized adopting [13] only renormalizable superpotential terms similar to those which lead to FHI. The $\mu$ parameter of MSSM can also be generated from the PQ scale as in Ref. [5]. On the other hand, this scheme may lead [14] to disastrous domain walls which, however, can be avoided [15, 16] by introducing extra matter superfields without jeopardizing the unification of the MSSM gauge coupling constants. Moreover, the structure of the superpotential obliges us to consider extra couplings between the inflaton field of FHI and the inflaton-like field of PQPT in the Kälher potential. As a bonus, these couplings assist us to reconcile the results on the (scalar) spectral index $n_{\mathrm{s}}$ of FHI and the recent seven-year results [17] from the Wilkinson microwave anisotropy probe (WMAP7) satellite.

Indeed, it is well-known that the realization of FHI within minimal Supergravity (SUGRA) leads to $n_{\mathrm{s}}$ which is just marginally consistent with the fitting of the WMAP7 data by the standard power-law cosmological model with cold dark matter and a cosmological constant $(\Lambda \mathrm{CDM})$. One possible resolution (for other proposals, see Refs. 18 22]) of this problem is 23, 24] the addition to the Kähler potential of a non-minimal quatric term of the inflaton field with a convenient choice of its sign. As a consequence, a negative mass term for the inflaton is generated. In the largest part of the parameter space, the inflationary potential acquires a local maximum and 
minimum. Then, FHI of the hilltop type 25] can occur as the inflaton rolls from this maximum down to smaller values. Therefore, $n_{\mathrm{s}}$ can become consistent with data, but only at the cost of an extra indispensable mild tuning [23] of the initial conditions. Another possible complication is that the system gets trapped near the minimum of the inflationary potential and, consequently, no FHI takes place.

Within our proposal, a negative mass term for the inflaton is generated too, which however depends on the various coefficients (of the order $0.01-0.1$ ) involved in the Kähler potential and not exclusively on the quatric term of the inflaton field. As a consequence, sufficiently low $n_{\mathrm{S}}$ can be achieved with either sign of the terms in the Kähler potential and the inflationary potential remains monotonic during the period of FHI. Therefore, complications due to the appearance of maxima and minima along the inflationary path can be eluded. Another byproduct of our proposal is that the reheat temperature after PQPT, calculated consistently with the model interactions, turns out to be low enough so that the gravitino $(\widetilde{G})$ constraint $[26,27]$ and the potential problem of topological defects 28] of FHI can be significantly relaxed since an entropy release takes place after PQPT. On the other hand, the reheat temperature is high enough so that non-perturbative electroweak sphalerons are operative and, consequently, thermal [29] or non-thermal [30] leptogenesis can, in principle, work.

Below, we present the basic ingredients of our model (Sec. III) and describe the inflationary potential and dynamics (Secs. III and IV). We then exhibit the constraints imposed on our cosmological set-up (Sec.V). We end up with our numerical results (Sec. (VI) and our conclusions (Sec. VII).

\section{MODEL DESCRIPTION}

\section{A. The general set-up}

In order to explore our scenario, we adopt the left-right symmetric gauge group $G_{\mathrm{LR}}=\mathrm{SU}(3)_{\mathrm{c}} \times$ $\mathrm{SU}(2)_{\mathrm{L}} \times \mathrm{SU}(2)_{\mathrm{R}} \times \mathrm{U}(1)_{B-L}$. In our scheme, $G_{\mathrm{LR}}$ can be broken down to the standard model gauge group $G_{\mathrm{SM}}$ at a scale close to the SUSY GUT scale $M_{\mathrm{GUT}}$ through the VEVs acquired by a conjugate pair of $\mathrm{SU}(2)_{\mathrm{R}}$ doublet left-handed Higgs superfields, $\bar{\Phi}$ and $\Phi$, with $B-L=$ $-1,1$ respectively. As a consequence, no cosmic strings are produced [28, 31] in this realization of standard FHI and, therefore, we are not obliged to impose extra restrictions on the parameters - as e.g. in Ref. 32].

The model possesses also three global U(1) symmetries. Namely, a (color) anomalous PQ symmetry $\mathrm{U}(1)_{\mathrm{PQ}}$, an anomalous $\mathrm{R}$ symmetry $\mathrm{U}(1)_{R}$, and the baryon number symmetry $\mathrm{U}(1)_{B}$. Note that global continuous symmetries can effectively arise [33] from the rich discrete symmetry groups encountered in many compactified string theories (see e.g. Ref. [34]). The PQ sym-
TABLE I: Superfield Content of the Model

\begin{tabular}{cccccc}
\hline \hline Superfields & Representations & \multicolumn{5}{c}{ Global Symmetries } \\
& under $G_{\mathrm{LR}}$ & $\mathrm{R}$ & $\mathrm{PQ}$ & $B$ & $D$ \\
\hline \multicolumn{7}{c}{ Matter Fields } & & & \\
\hline$l_{i}$ & $(\mathbf{1}, \mathbf{2}, \mathbf{1}, \mathbf{- 1})$ & 0 & -2 & 0 & 0 \\
$l_{i}^{c}$ & $(\mathbf{1}, \mathbf{1}, \mathbf{2}, \mathbf{1})$ & 2 & 0 & 0 & 0 \\
$q_{i}$ & $(\mathbf{3}, \mathbf{2}, \mathbf{1}, \mathbf{1} / \mathbf{3})$ & 1 & -1 & $1 / 3$ & 0 \\
$q_{i}^{c}$ & $(\overline{\mathbf{3}}, \mathbf{1}, \mathbf{2}, \mathbf{- 1} / \mathbf{3})$ & 1 & -1 & $-1 / 3$ & 0 \\
\hline \multicolumn{7}{c}{ Higgs Fields } & & & \\
\hline$S$ & $(\mathbf{1}, \mathbf{1}, \mathbf{1}, \mathbf{0})$ & 4 & 0 & 0 & 0 \\
$\bar{\Phi}$ & $(\mathbf{1}, \mathbf{1}, \mathbf{2},-\mathbf{1})$ & 0 & 0 & 0 & 0 \\
$\Phi$ & $(\mathbf{1}, \mathbf{1}, \mathbf{2}, \mathbf{1})$ & 0 & 0 & 0 & 0 \\
\hline$P$ & $(\mathbf{1}, \mathbf{1}, \mathbf{1}, \mathbf{0})$ & 4 & 0 & 0 & 0 \\
$\bar{Q}$ & $(\mathbf{1}, \mathbf{1}, \mathbf{1}, \mathbf{0})$ & 0 & -2 & 0 & 0 \\
$Q$ & $(\mathbf{1}, \mathbf{1}, \mathbf{1}, \mathbf{0})$ & 0 & 2 & 0 & 0 \\
\hline$h$ & $(\mathbf{1}, \mathbf{2}, \mathbf{2}, \mathbf{0})$ & 2 & 2 & 0 & 0 \\
\hline $\bar{D}_{\mathrm{a}}$ & Extra Matter Fields & & \\
\hline$D_{\mathrm{a}}$ & $(\overline{\mathbf{3}}, \mathbf{1}, \mathbf{1}, \mathbf{2} / \mathbf{3})$ & 2 & 1 & 0 & -1 \\
$H_{\mathrm{a}}$ & $(\mathbf{3}, \mathbf{1}, \mathbf{1}, \mathbf{- 2} / \mathbf{3})$ & 2 & 1 & 0 & 1 \\
\hline \hline
\end{tabular}

metry $\mathrm{U}(1)_{\mathrm{PQ}}$ can be spontaneously broken at the $\mathrm{PQ}$ breaking scale $f_{a} \sim\left(10^{10}-10^{12}\right) \mathrm{GeV}$ (which coincides with the axion decay constant - for a review see Ref. 35]) via the VEVs acquired by two $G_{\mathrm{LR}}$ singlet left-handed superfields $\bar{Q}$ and $Q$.

The part of the superpotential which is relevant for the breaking of the group $G_{\mathrm{LR}} \times \mathrm{U}(1)_{\mathrm{PQ}}$ is

$$
W=\kappa S\left(\bar{\Phi} \Phi-M^{2}\right)+\kappa_{a} P\left(\bar{Q} Q-f_{a}^{2} / 4\right)+\lambda S \bar{Q} Q,
$$

where $S, P$ are $G_{\mathrm{LR}}$ singlet left-handed superfields which trigger the breaking of $G_{\mathrm{LR}}$ and $\mathrm{U}(1)_{\mathrm{PQ}}$ respectively and the parameters $\kappa, \kappa_{a}, M \sim M_{\mathrm{GUT}}$, and $f_{a}$ are made positive by field redefinitions. For simplicity, we restrict our analysis to real $\lambda$ 's. The superfield $P$ can be regarded as the linear combination of the $G_{\mathrm{LR}}$ singlets with $\mathrm{PQ}$ and $\mathrm{R}$ charge equal to 0 and 4 respectively that does not couple to $\bar{\Phi} \Phi-$ cf. Ref. [36]. In this basis, the most general Kähler potential of our model includes interference terms of $S$ and $P$ even at the quadratic level - contrary to the choice opted in Ref. 37]. Namely, we adopt the following Kähler potential

$$
\begin{aligned}
K= & |S|^{2}+|P|^{2}+\mathrm{a}\left(S P^{*}+S^{*} P\right) \\
& +b \frac{|S|^{4}}{4 m_{\mathrm{P}}^{2}}+c \frac{|P|^{4}}{4 m_{\mathrm{P}}^{2}}+d \frac{|S|^{2}|P|^{2}}{m_{\mathrm{P}}^{2}} \\
& +\frac{e|S|^{2}+f|P|^{2}}{2 m_{\mathrm{P}}^{2}}\left(S P^{*}+S^{*} P\right) \\
& +\frac{g}{4 m_{\mathrm{P}}^{2}}\left[\left(S P^{*}\right)^{2}+\left(S^{*} P\right)^{2}\right] \\
& +|\Phi|^{2}+|\bar{\Phi}|^{2}+|Q|^{2}+|\bar{Q}|^{2} \\
& +k\left(\Phi \bar{\Phi}+\Phi^{*} \bar{\Phi}^{*}\right)+l\left(Q \bar{Q}+Q^{*} \bar{Q}^{*}\right)+\cdots,
\end{aligned}
$$


where all the coefficients a, $b, c, d, e, f, g, k$, and $l$ are taken, for simplicity, real although some of them can be, in general, complex. The ellipsis represents higher order terms involving the waterfall fields $(\Phi, \bar{\Phi}, Q$, and $\bar{Q})$ and $S$ and $P$. We can neglect these terms since they have negligible impact on the SUSY vacuum and are irrelevant along the inflationary path - see below.

The usual superpotential terms of MSSM can be derived from the following superpotential:

$$
W_{\mathrm{m}}=\lambda_{\mu} \frac{\bar{Q}^{2} h^{2}}{2 m_{\mathrm{P}}}+y_{\nu i j} \frac{\bar{\Phi} l_{i}^{c} \bar{\Phi} l_{j}^{c}}{m_{\mathrm{P}}}+y_{l i j} l_{i} h l_{j}^{c}+y_{q i j} q_{i} h q_{j}^{c}
$$

with $m_{\mathrm{P}} \simeq 2.44 \times 10^{18} \mathrm{GeV}$ being the reduced Planck scale. Here, the $i$ th generation $\mathrm{SU}(2)_{\mathrm{L}}$ doublet lefthanded quark and lepton superfields are denoted by $q_{i}$ and $l_{i}$ respectively, whereas the $\mathrm{SU}(2)_{\mathrm{R}}$ doublet antiquark and antilepton superfields by $q_{i}^{c}$ and $l_{i}^{c}$ respectively. The electroweak Higgs superfields are contained in a $\mathrm{SU}(2)_{\mathrm{L}} \times \mathrm{SU}(2)_{\mathrm{R}}$ bidoublet Higgs superfield $h$. The first term in the right-hand side (RHS) of Eq. (3) generates the $\mu$ term of MSSM via the PQ breaking scale (see below), while the second term generates intermediate scale masses for the right-handed neutrinos and, thus, seesaw masses [2] for the light neutrinos.

The representations under $G_{\mathrm{LR}}$ and the charges under the global symmetries of the various matter and Higgs superfields contained in this model are presented in Table I. which also contains the extra matter superfields required for evading the domain wall problem associated with the PQPT together with a new imposed global symmetry $\mathrm{U}(1)_{D}-$ see Sec. IIC.

\section{B. The cosmological scenario}

The F-term SUGRA scalar potential obtained from $W$ in Eq. (11) and $K$ in Eq. (2) can be found by applying the well-known formula (see e.g. Ref. [1])

$$
V_{\mathrm{SUGRA}}=e^{K / m_{\mathrm{P}}^{2}}\left(F_{i^{*}}^{*}\left(K_{, j i^{*}}\right)^{-1} F_{j}-3 \frac{|W|^{2}}{m_{\mathrm{P}}^{2}}\right),
$$

where $F_{i}=W_{, i}+K_{, i} W / m_{\mathrm{P}}^{2}$ and a subscript , $i\left[i^{*}\right]$ denotes derivation with respect to (w.r.t.) the complex scalar field $i\left[i^{*}\right]$. In the limit where $m_{\mathrm{P}}$ tends to infinity, we can obtain the SUSY limit, $V_{\mathrm{F}}$, of $V_{\mathrm{SUGRA}}$ in Eq. (4) which turns out to be

$$
\begin{aligned}
V_{\mathrm{F}} \simeq & \left|\kappa\left(\bar{\Phi} \Phi-M^{2}\right)+\lambda \bar{Q} Q\right|^{2} /\left(1-\mathrm{a}^{2}\right) \\
& +\kappa_{a}^{2}\left|\bar{Q} Q-f_{a}^{2} / 4\right|^{2} /\left(1-\mathrm{a}^{2}\right) \\
& +\kappa^{2}|S|^{2}\left(|\bar{\Phi}|^{2}+|\Phi|^{2}\right) \\
& +\left|\lambda S+\kappa_{a} P\right|^{2}\left(|\bar{Q}|^{2}+|Q|^{2}\right) \\
& -\left[a \kappa _ { a } ( \overline { Q } ^ { * } Q ^ { * } - f _ { a } ^ { 2 } / 4 ) \left[\kappa\left(\bar{\Phi} \Phi-M^{2}\right)\right.\right. \\
& \left.+\lambda \bar{Q} Q] /\left(1-\mathrm{a}^{2}\right)+\text { complex conjugate }\right]
\end{aligned}
$$

where the complex scalar fields which belong to the SM singlet components of the superfields are denoted by the same symbol and we take into account that $M \gg f_{a}$. Note that although the mixing terms in the first line of Eq. (2) have an impact on Eq. (5), the corresponding terms in the last line of this equation do not contribute since they are holomorphic or anti-holomorphic in the field variables. Also the terms in the ellipsis in Eq. (2) have no appreciable contribution to the RHS of Eq. (5) since they are suppressed by powers of $m_{\mathrm{P}}$.

The D-term contribution vanishes along the direction

$$
|\bar{\Phi}|=|\Phi| .
$$

From the potential in Eq. (5) and taking into account that $M \gg f_{a}$, we find that the SUSY vacuum lies at

$$
\langle S\rangle=0,|\langle\bar{\Phi}\rangle|=|\langle\Phi\rangle| \simeq M,\langle P\rangle=0 \text {, and }\left|\left\langle\phi_{Q}\right\rangle\right|=f_{a}
$$

where we have introduced the canonically normalized scalar field $\phi_{Q}=2 Q=2 \bar{Q}$. Note that, since the sum of the arguments of $\langle\bar{Q}\rangle,\langle Q\rangle$ must be $0, \bar{Q}$ and $Q$ can be brought to the real axis by an appropriate PQ transformation. As a consequence of Eqs. (7), $W$ leads to a spontaneous breaking of $G_{\mathrm{LR}}$ and $\mathrm{U}_{\mathrm{PQ}}(1)$. The same superpotential $W$ gives also rise to a stage of $\mathrm{FHI}$ and a PQPT, which is highlighted in the following.

The potential in Eq. (5) possesses a D- and F-flat direction at

$$
\bar{\Phi}=\Phi=0 \text { and } \bar{Q}=Q=0
$$

with a constant potential energy density

$$
\left.V_{\mathrm{HI} 0} \simeq \frac{\kappa^{2} M^{4}}{1-\mathrm{a}^{2}} \quad \text { (since } M \gg f_{a}\right) .
$$

The direction in Eq. (8) can be used as the inflationary trajectory since it corresponds to a classically flat valley of minima for

$$
\text { (a) }|S|>\frac{M}{\sqrt{1-\mathrm{a}^{2}}} \text { and (b) }\left|\sigma_{a}\right|>\sqrt{\frac{\kappa\left(\lambda-\mathrm{a} \kappa_{a}\right)}{1-\mathrm{a}^{2}}} M \text {, }
$$

where we have defined $\sigma_{a}=\lambda S+\kappa_{a} P$.

For relatively large $\lambda$ 's, the reheating temperature after FHI turns out to be rather high, leading to troubles with the $\widetilde{G}$ abundance [26, 27] - as usually in SUSY cosmological models. However, we can verify that a D- and F-flat direction appears at

$$
S=0, \bar{\Phi}=\Phi=M, \text { and } \bar{Q}=Q=0
$$

with potential energy density

$$
V_{\mathrm{PQ} 0}=\kappa_{a}^{2} f_{a}^{4} / 16 .
$$

Since $V_{\mathrm{PQ0}} \ll V_{\mathrm{HI}}, V_{\mathrm{PQ} 0}$ can temporally dominate over radiation after the end of $\mathrm{FHI}$ and, if $|P| \geq f_{a}$, drive a PQPT - see Sec. IV A subsequent second episode of 
TABLE II: The mass spectrum of the model along the inflationary trajectory of Eqs. (8) and (10).

\begin{tabular}{c|c|c}
\hline $\begin{array}{c}\text { Superfields } \\
\text { of origin }\end{array}$ & Fields & Mass Squared \\
\hline \multicolumn{3}{c}{ Bosons } \\
\hline $\bar{\Phi}, \Phi$ & 4 complex scalars & $\kappa^{2}\left(|S|^{2} \pm \frac{M^{2}}{1-a^{2}}\right)$ \\
$\bar{Q}, Q$ & 2 complex scalars & $\left|\sigma_{a}\right|^{2} \pm \frac{\kappa\left(\lambda-a \kappa_{a}\right) M^{2}}{1-a^{2}}$ \\
\hline \multicolumn{3}{c}{ Fermions } \\
\hline $\bar{\Phi}, \Phi$ & 2 Dirac spinors & $\kappa^{2}|S|^{2}$ \\
$\bar{Q}, Q$ & 1 Dirac spinors & $\left|\sigma_{a}\right|^{2}$ \\
\hline \hline
\end{tabular}

reheating can dilute sufficiently the unwanted $\widetilde{G}$ concentration - see Appendix A. At the end of PQPT, the fields $P, \bar{Q}$, and $Q$ acquire their VEVs in Eq. (7). The $\mu$ term of the MSSM is generated via the first term in the RHS of Eq. (3) with $|\mu| \sim \lambda_{\mu}|\langle\bar{Q}\rangle|^{2} / m_{\mathrm{P}}$, which is of the right magnitude if $|\langle\bar{Q}\rangle|=f_{a} / 2 \simeq 5 \times 10^{11} \mathrm{GeV}$ and $\lambda_{\mu} \sim(0.001-0.01)$.

The cosmological scenario above can be attained if we ensure that, at the end of FHI, the fields $\bar{\Phi}$ and $\Phi$ acquire their VEVs in Eq. (7), while the fields $\bar{Q}$ and $Q$ remain equal to zero. To quantify this crucial requirement, we construct the mass spectrum of the theory along the inflationary path of Eqs. (8) and (10). We summarize our results in Table II. We see that the mass matrices of the scalar components of the $\bar{\Phi}, \Phi$ and $\bar{Q}, Q$ superfields develop a negative eigenvalue as $|S|$ and $\left|\sigma_{a}\right|$ cross below their critical values - i.e. their lower bounds in Eq. (10). However, if the tachyonic instability of the $\bar{\Phi}-\Phi$ system occurs first, $\bar{\Phi}$ and $\Phi$ start evolving towards their VEVs, whereas $\bar{Q}$ and $Q$ continue to be confined to zero.

\section{Evading the domain-wall problem}

It should be mentioned that instanton and soft SUSY breaking effects explicitly break $\mathrm{U}(1)_{R} \times \mathrm{U}(1)_{\mathrm{PQ}}$ to a discrete subgroup. Spontaneous breaking of this subgroup at the PQPT by $\langle\bar{Q}\rangle$ and $\langle Q\rangle$ can lead [14] to a disastrous domain wall production since this transition occurs after FHI. In order to avoid this problem, we must introduce some extra matter fields (see Table (I) following Ref. [15] (see also Ref. [16], where the set-up is similar to ours). Namely, we introduce $n$ pairs of left-handed superfields $\bar{D}_{\mathrm{a}}$ and $D_{\mathrm{a}}(\mathrm{a}=1, \ldots, n)$ which are $\mathrm{SU}(3)_{\mathrm{c}}$ antitriplets and triplets respectively with $\mathrm{R}$ charge equal to 2 and $\mathrm{PQ}$ charge equal to 1 . However, these fields acquire intermediate scale masses after the PQ breaking, which could prevent the unification of the MSSM gauge coupling constants. To restore gauge unification, we include an equal number of $\mathrm{SU}(2)_{\mathrm{L}} \times \mathrm{SU}(2)_{\mathrm{R}}$ bidoublet superfields $H_{\mathrm{a}}$ with $\mathrm{PQ}$ and $\mathrm{R}$ charges equal to those of $\bar{D}_{\mathrm{a}}$ and $D_{\mathrm{a}}$. In accordance with all the imposed symmetries, we can give intermediate scale masses to $\bar{D}_{\mathrm{a}}-D_{\mathrm{a}}$ and
$H_{\mathrm{a}}$ through the superpotential terms

$$
W_{\mathrm{dw}}=\lambda_{D \mathrm{a}} \bar{Q} \bar{D}_{\mathrm{a}} D_{\mathrm{a}}+\lambda_{H \mathrm{a}} \bar{Q} H_{\mathrm{a}}^{2}
$$

Here, we chose a basis in the $\bar{D}_{\mathrm{a}}-D_{\mathrm{a}}$ and $H_{\mathrm{a}}$ space where the coupling constant matrices $\lambda_{D \mathrm{a}}$ and $\lambda_{H \mathrm{a}}$ are diagonal. Note that the full superpotential is invariant under a new global U $(1)_{D}$ symmetry - see Table I. During FHI, we take $\bar{D}_{\mathrm{a}}=D_{\mathrm{a}}=H_{\mathrm{a}}=0$ and, at the SUSY vacuum, we have $\left\langle\bar{D}_{\mathrm{a}}\right\rangle=\left\langle D_{\mathrm{a}}\right\rangle=\left\langle H_{\mathrm{a}}\right\rangle=0$.

The number $n$ can be determined as follows: The explicitly unbroken subgroup of $\mathrm{U}(1)_{R} \times \mathrm{U}(1)_{\mathrm{PQ}}$ can be found, for every $n$, from the solutions of the system

$4 \alpha=0(\bmod 2 \pi)$ and $-12 \alpha+2(n-6) \beta=0(\bmod 2 \pi)$,

where $\alpha$ and $\beta$ are the phases of a $\mathrm{U}(1)_{R}$ and $\mathrm{U}(1)_{\mathrm{PQ}}$ rotation respectively. Here we took into account that the $\mathrm{R}$ charge of $W$ and, thus, of all the soft SUSY breaking term is 4 and that the sum of the $\mathrm{R}[\mathrm{PQ}]$ charges of the $\mathrm{SU}(3)_{\mathrm{c}}$ triplets and antitriplets is $-12[2(n-6)]$. We can induce, therefore, that the explicitly unbroken subgroup is $Z_{4} \times Z_{2(n-6)}$. It is then important to ensure that this subgroup is not spontaneously broken by $\langle\bar{Q}\rangle$ and $\langle Q\rangle$, i.e.,

$$
2 \beta=0(\bmod 2 \pi)
$$

since otherwise cosmologically disastrous domain walls will be produced at PQPT. This requirement implies that $n$ must be 5 or 7 . In both these cases, the subgroup of $\mathrm{U}(1)_{R} \times \mathrm{U}(1)_{\mathrm{PQ}}$ left unbroken by instantons and SUSY breaking coincides with the one left unbroken by $\langle\bar{Q}\rangle$ and $\langle Q\rangle$ and is a $Z_{4} \times Z_{2}$.

It is easy to find the contribution of $\bar{D}_{\mathrm{a}}, D_{\mathrm{a}}$, and $H_{\mathrm{a}}$ to the coefficients $b_{1}, b_{2}$, and $b_{3}$ controlling [38] the one loop evolution of the three gauge coupling constants $g_{1}, g_{2}$, and $g_{3}$ within MSSM. It is then straightforward to prove that, if we assign $B-L=2 / 3$ and $-2 / 3$ to $\bar{D}_{\text {a }}$ and $D_{\text {a }}$ respectively, the quantities $b_{2}-b_{1}$ and $b_{3}-b_{2}$ (which are [38] crucial for the unification of $g_{1}, g_{2}$, and $g_{3}$ ) remain unaltered. Therefore, the inclusion of the extra matter superfields does not disturb the gauge unification at one loop.

Recapitulating this section, let us present the total superpotential of our model which is

$$
W_{\mathrm{tot}}=W+W_{\mathrm{m}}+W_{\mathrm{dw}},
$$

where $W, W_{\mathrm{m}}$ and $W_{\mathrm{dw}}$ are given in Eq. (11), (3), and (13) respectively.

\section{THE INFLATIONARY EPOCH}

\section{A. Structure of the inflationary potential}

The potential which can drive the inflationary stage of our set-up has the following general form

$$
V_{\mathrm{HI}}=V_{\mathrm{HIO}}+V_{\mathrm{HIs}}+V_{\mathrm{HIc}},
$$


where $V_{\text {HIs }}$ and $V_{\text {HIc }}$ represent, respectively, SUGRA and one-loop radiative corrections to the inflationary potential, calculated in Sec. III A 1 and III A 2, Let us note, in passing, that the most important contribution [39] to $V_{\mathrm{HI}}$ from the soft SUSY breaking terms is expected to start playing an important role for rather small $\kappa$ 's and so, it remains negligibly small in our set-up due to the large $\kappa$ 's used - see Sec. VI

\section{Supergravity corrections}

Expanding the F-term SUGRA potential in Eq. (4) along the inflationary trajectory - see Eq. (8) - in powers of $1 / m_{\mathrm{P}}$ for $M \gg f_{a}$, we obtain the following expression:

$$
\begin{aligned}
V_{\mathrm{HIS}} \simeq & \frac{V_{\mathrm{HI} 0}}{\left(1-\mathrm{a}^{2}\right) m_{\mathrm{P}}^{2}}\left[A_{1}|S|^{2}+A_{12}\left(S^{*} P+P S^{*}\right)+A_{2}|P|^{2}\right]+\frac{V_{\mathrm{HI} 0}}{4\left(1-\mathrm{a}^{2}\right)^{2} m_{\mathrm{P}}^{4}}\left[B_{1}|S|^{4}+B_{2}|P|^{4}\right. \\
& \left.+B_{3}|S|^{2}|P|^{2}+\left(B_{4}|S|^{2}+B_{5}|P|^{2}\right)\left(S^{*} P+P S^{*}\right)+B_{6}\left(\left(S^{*} P\right)^{2}+\left(P^{*} S\right)^{2}\right)\right]
\end{aligned}
$$

where

$$
\begin{aligned}
A_{1}= & 2 \mathrm{a} e-\mathrm{a}^{4}-\mathrm{a}^{2}(d-1)-b, A_{2}=1-d-\mathrm{a}(\mathrm{a}+\mathrm{a} c-2 f), A_{12}=\mathrm{a}(1+d-\mathrm{a}(\mathrm{a}+f)+g)-e, \\
B_{1}= & 2+\mathrm{a}^{4}(2+4 d-3 b)+b(4 b-7)+4 \mathrm{a}^{5} e-8 \mathrm{a}^{3}(2+d) e-4 \mathrm{a}(2 d+4 b-3) e+4 e^{2} \\
& +2 \mathrm{a}^{2}\left(5 b+2 d(d+2 b-1)+6 e^{2}-2\right), \\
B_{2}= & 2+c+\mathrm{a}^{4}(5 c+2)+4(d-1) d-8 \mathrm{a}^{3}(c+1) f-8 \mathrm{a}(c+2 d-1) f+4 f^{2}+2 \mathrm{a}^{2}\left((2 c+1)(c+2 d-2)+6 f^{2}\right),(21) \\
B_{3}= & 4\left[1+\mathrm{a}^{6}+(d-2) d+b(2 d-1)+4 \mathrm{a}^{5} f+2 e(e+f)-2 \mathrm{a}(c e+2(b-1) f+(2 e+f)(2 d+g))+g^{2}\right. \\
& +\mathrm{a}^{4}(c-2(d+g)-1)-2 \mathrm{a}^{3}(c e+f(4+2 d+g))+\mathrm{a}^{2}(b(1+2 c)+4 d+c(2 d-1)+2 f(5 e+f) \\
& +2 g+(d+g)(3 d+g)-1)] \\
B_{4}= & 2\left[2 \mathrm{a}^{4}(f-3 e)+2 e(2 b+d+g-2)+\mathrm{a}^{5}(2+4 d+g)+2 \mathrm{a}^{3}(b-d(4+d+g)-2(1+e f+g))\right. \\
& \left.+2 \mathrm{a}^{2}((2 b+2 d-1) f+e(5+5 d+3 g))-\mathrm{a}(4 e(2 e+f)-3 g+2 d(d+g-2)+2 b(2 d+2 g+1)-2)\right], \\
B_{5}= & 2\left[2((2 d-1) e+f(d+g))-2 \mathrm{a}^{4} f-2 \mathrm{a}^{3}\left(2 f^{2}+g+c(d+g+2)+2\right)+2 \mathrm{a}^{2}(e+2 c e+f(2 c+5 d+3 g+1))\right. \\
& \left.+\mathrm{a}^{5}(2+3 c)+\mathrm{a}(1-2 d-2 g)+2 \mathrm{a}(1-2 f(2 e+f)+g-2 d(d+g))\right],
\end{aligned}
$$

and

$$
\begin{aligned}
B_{6}= & 2 \mathrm{a}^{6}+4 e^{2}+6 \mathrm{a}^{5} f+(4 d-1) g-\mathrm{a}^{4}(4+4 d+5 g)+4 \mathrm{a}^{3}(e-f(2+d+g))-2 \mathrm{a}(f(2 d+2 g-1) \\
& +e(2+4 d+4 g))+2 \mathrm{a}^{2}\left(2 f(2 e+f)+3 g+2\left(d+d^{2}+g d+g^{2}\right)+1\right) .
\end{aligned}
$$

In this expansion, we neglect terms proportional to $\sqrt{V_{\mathrm{HI} 0} V_{\mathrm{PQ0}}} / m_{\mathrm{P}}^{2}$, which are numerically suppressed. To specify the canonically normalized scalar field, which could play the role of inflaton, we have firstly to bring into a canonical form, through a non-unitary transformation, the quadratic part $K_{S P}$ of the Kähler potential in Eq. (2) which involves $S$ and $P$. Namely, we find

$$
K_{S P}=|S|^{2}+|P|^{2}+\mathrm{a}\left(S P^{*}+S^{*} P\right)=\left|s_{+}\right|^{2}+\left|s_{-}\right|^{2},
$$

where $s_{ \pm}=A_{ \pm}(S \pm P) / \sqrt{2}$ with $A_{ \pm}=\sqrt{1 \pm a}$. Solving w.r.t. $S$ and $P$, we get

$$
S=\frac{1}{\sqrt{2}}\left(\frac{s_{+}}{A_{+}}+\frac{s_{-}}{A_{-}}\right) \text {and } P=\frac{1}{\sqrt{2}}\left(\frac{s_{+}}{A_{+}}-\frac{s_{-}}{A_{-}}\right) .
$$

Introducing the real and imaginary components of the fields $s_{ \pm}$from the relations $s_{ \pm}=\left(s_{1 \pm}+i s_{2 \pm}\right) / \sqrt{2}$, the first term of $V_{\mathrm{HIs}}$ in Eq. (18) reads

$$
\frac{V_{\mathrm{HIO}}}{2 m_{\mathrm{P}}^{2}}\left(\left(\begin{array}{ll}
s_{1-} & s_{1+}
\end{array}\right) M_{1}^{2}\left(\begin{array}{c}
s_{1-} \\
s_{1+}
\end{array}\right)+(1 \leftrightarrow 2)\right),
$$

where the matrices $M_{1}^{2}$ and $M_{2}^{2}$ are found to be

$$
M_{1}^{2}=M_{2}^{2}=\left(\begin{array}{cc}
\frac{A_{1}+A_{2}-2 A_{12}}{(1-a)^{2}(1+a)} & \frac{A_{1}-A_{2}}{\left(1-a^{2}\right)^{3 / 2}} \\
\frac{A_{1}-A_{2}}{\left(1-a^{2}\right)^{3 / 2}} & \frac{A_{1}+A_{2}+2 A_{12}}{(1+a)^{2}(1-a)}
\end{array}\right) .
$$

To identify the combination of $s_{ \pm}$which can play the role of inflaton, we have to diagonalize the matrices $M_{1}^{2}$ and $M_{2}^{2}$. This can be realized via an orthogonal matrix $U$ as follows ( $\mathrm{T}$ stands for the transpose of a matrix):

$$
U M_{1}^{2} U^{\top}=U M_{2}^{2} U^{\top}=\operatorname{diag}\left(m_{+}^{2}, m_{-}^{2}\right),
$$

where

$$
m_{ \pm}^{2}=\frac{D_{1} \pm \sqrt{D_{2}}}{\left(1-\mathrm{a}^{2}\right)^{2}}
$$


with

$$
D_{1}=A_{1}-2 \mathrm{a} A_{12}+A_{2}
$$

and

$$
D_{2}=D_{1}^{2}+4\left(1-\mathrm{a}^{2}\right)\left(A_{12}^{2}-A_{1} A_{2}\right) .
$$

Also

$$
U=\left(\begin{array}{cc}
1 / N_{+} & 1 / N_{-} \\
U_{+} / N_{+} & U_{-} / N_{-}
\end{array}\right)
$$

with

$$
U_{ \pm}=\frac{\mathrm{a} A_{1}-2 A_{12}+\mathrm{a} A_{2} \pm \sqrt{D_{2}}}{\sqrt{1-\mathrm{a}^{2}}\left(A_{1}-A_{2}\right)} \text { and } N_{ \pm}=\sqrt{1+U_{ \pm}^{2}} .
$$

Embedding unity $\left(1=U U^{\top}=U^{\top} U\right)$ on the left and the right of $M_{1}^{2}$ and $M_{2}^{2}$ in Eq. (28), this expression can be brought into the form

$$
\frac{V_{\mathrm{HI} 0}}{m_{\mathrm{P}}^{2}}\left(m_{+}^{2}\left|\sigma_{+}\right|^{2}+m_{-}^{2}\left|\sigma_{-}\right|^{2}\right),
$$

where the real and imaginary components of the complex fields $\sigma_{+}$and $\sigma_{-}$, defined as $\sigma_{ \pm}=\left(\sigma_{1 \pm}+i \sigma_{2 \pm}\right) / \sqrt{2}$, are given by

$$
\begin{aligned}
& \sigma_{1-}=s_{1-} / N_{+}+s_{1+} / N_{-} \text {and }(1 \leftrightarrow 2), \\
& \sigma_{1+}=s_{1-} U_{+} / N_{+}+s_{1+} U_{-} / N_{-} \text {and }(1 \leftrightarrow 2) .
\end{aligned}
$$

Performing an appropriate $\mathrm{R}$ transformation, we can rotate $\sigma_{-}$to the real axis (setting $\sigma_{2-}=0$ ). To simplify the notation, we rename the remaining fields as follows: $\sigma_{1-}=\sigma, \quad \sigma_{1+}=s$ and $\sigma_{2+}=q$. We then can solve Eqs. (37) and (38) w.r.t. the components of $s_{ \pm}$, i.e.,

$$
\begin{aligned}
& s_{1-}=N_{+} \frac{s-U_{-} \sigma}{U_{+}-U_{-}} \text {and } s_{2-}=\frac{N_{+} q}{U_{+}-U_{-}} \\
& s_{1+}=N_{-} \frac{s-U_{+} \sigma}{U_{-}-U_{+}} \text {and } s_{2+}=\frac{N_{-} q}{U_{-}-U_{+}} .
\end{aligned}
$$

Substituting the expressions above into Eq. (27) and then into Eq. (18), we can derive $V_{\mathrm{HIs}}$ as a function of $\sigma, s$, and $q$. As can be explicitly verified, $K_{S P}$ remains canonical w.r.t the latter fields - i.e. $K_{S P}=\left(\sigma^{2}+s^{2}+q^{2}\right) / 2-$ since the $U$ transformation, which connects $\sigma, s$, and $q$ with $s_{1 \pm}$ and $s_{2 \pm}$, is orthogonal.

From the expressions above, we easily conclude that, if we set $b=c=d=e=f=g=0$ with $a \neq 0$, we take

$$
\left(\frac{A_{1}}{1-\mathrm{a}^{2}}, \frac{A_{12}}{1-\mathrm{a}^{2}}, \frac{A_{2}}{1-\mathrm{a}^{2}}\right)=\left(\mathrm{a}^{2}, \mathrm{a}, 1\right)
$$

which results to $m_{-}^{2}=0$ and $m_{+}^{2}=2$ via Eq. (31). In other words, in this case, $\sigma$ remains identically massless, whereas $s$ and $q$ acquire effective masses equal to $\sqrt{3 /\left(1-a^{2}\right)} H_{\mathrm{HI} 0}$ with $H_{\mathrm{HI} O}=\sqrt{V_{\mathrm{HI}}} / \sqrt{3} m_{\mathrm{P}}$. Therefore, we are obliged to invoke non-zero coefficients of the higher order terms of the Kähler potential considered in
Eq. (2), in order to generate a negative $m_{-}^{2}$, and to reduce, thereby, $n_{\mathrm{s}}$ to an observationally acceptable level. Needless to say that, for $a=b=c=d=e=f=g=0$, the well-known results (see e.g. Refs 40, 41]) in the context of minimal SUGRA can be obviously recovered - note that, in this case, $B_{1}=B_{2}=B_{3} / 2=2$ and $B_{4}=B_{5}=B_{6}=0$.

\section{Radiative corrections}

The constant tree-level potential energy density $V_{\mathrm{HIO}}$ causes SUSY breaking leading [2] to the generation of one-loop radiative corrections, which provide a logarithmic slope along the inflationary path necessary for driving the system towards the vacua. Inserting the spectrum shown in Table $\amalg$ in the well-known Coleman-Weinberg formula [42], we find (compare with Ref. 36] ) that the one-loop radiative correction to $V_{\mathrm{HI}}$ is

$$
V_{\mathrm{HIc}}=V_{\kappa}+V_{\lambda} \text {, }
$$

where

$$
V_{\kappa}=\frac{\kappa^{2} V_{\mathrm{HI} 0}}{16 \pi^{2}\left(1-\mathrm{a}^{2}\right)}\left(2 \ln \frac{\kappa^{2} x M^{2}}{\left(1-\mathrm{a}^{2}\right) \Lambda^{2}}+f_{\mathrm{rc}}(x)\right)
$$

with $x=\left(1-\mathrm{a}^{2}\right)|S|^{2} / M^{2}$ and

$V_{\lambda}=\frac{\left(\lambda-a \kappa_{a}\right)^{2} V_{\mathrm{HIO}}}{32 \pi^{2}\left(1-\mathrm{a}^{2}\right)}\left(2 \ln \frac{\kappa\left(\lambda-\mathrm{a} \kappa_{a}\right) x_{a} M^{2}}{\left(1-\mathrm{a}^{2}\right) \Lambda^{2}}+f_{\mathrm{rc}}\left(x_{a}\right)\right)$

with $x_{a}=\left(1-\mathrm{a}^{2}\right)\left|\sigma_{a}\right|^{2} / \kappa\left(\lambda-\mathrm{a} \kappa_{a}\right) M^{2}$. In the relations above, we have taken into account that the dimensionality of the representations to which $\bar{\Phi}$ and $\Phi[\bar{Q}$ and $Q]$ belong is $2[1]$ - see Table I - and we have defined

$$
f_{\mathrm{rc}}(y)=(y+1)^{2} \ln (1+1 / y)+(y-1)^{2} \ln (1-1 / y) .
$$

Let us note that, for $x \gg 1$ and $x_{a} \gg 1, V_{\kappa}\left[V_{\lambda}\right]$ can be well approximated by replacing $f_{\mathrm{rc}}(y) \simeq 3$ in the RHS of Eq. (43) [Eq. (44)] - see Sec. IIIB, Although rather large $\kappa$ 's, $\kappa_{a}$ 's, and $\lambda$ 's are used in our work, renormalization group effects [43] remain negligible and so our results are independent from the renormalization scale $\Lambda$.

Substituting Eqs. (39) and (40) into Eq. (27) and then into Eqs. (43) and (44), we can derive $V_{\mathrm{HIc}}$ as a function of $\sigma, s$, and $q$.

\section{B. The inflationary dynamics}

The evolution of the various fields involved in our scheme during FHI is governed by their equations of motion (e.o.m.)

$$
\ddot{f}+3 H \dot{f}+V_{\mathrm{HI}, f}=0 \text { with } f=\sigma, s, \text { and } q .
$$

where the dot denotes derivation w.r.t. the cosmic time $t$ and $H$ is the Hubble parameter. The solution of the 
system in Eq. (46) can be facilitated if we use as independent variable the number of e-foldings $N$ defined by

$$
N=\ln \left(R / R_{\mathrm{HIi}}\right) \Rightarrow \dot{N}=H \text { and } \dot{H}=H^{\prime} H
$$

with $R(t)$ being the scale factor of the universe and $R_{\mathrm{HIi}}$ its value at the commencement of FHI. Here the prime denotes derivation w.r.t. $N$. Converting the time derivatives to derivatives w.r.t. $N$, Eq. (46) becomes

$$
H^{2} f^{\prime \prime}+\left(3 H+H^{\prime}\right) H f^{\prime}+V_{\mathrm{HI}, f}=0
$$

with $f=\sigma, s$, or $q$. This system can be solved numerically for the period of FHI by taking

$$
H=H_{\mathrm{HI}}=\sqrt{\frac{2 V_{\mathrm{HI}}}{6 m_{\mathrm{P}}^{2}-\sigma^{\prime 2}-s^{\prime 2}-q^{\prime 2}}},
$$

$V=V_{\mathrm{HI}}$ given by Eq. (17), and imposing the following initial conditions (at $N=0$ ):

$$
f(0)=f_{\mathrm{HIi}} \text { and } f^{\prime}(0)=0 \text { with } f=\sigma, s, \text { or } q,
$$

where $f_{\mathrm{HIi}}$ is taken to be in the range $(1.5-4.5) \times$ $10^{17} \mathrm{GeV}$. We checked that our results are pretty stable against variation of $f_{\mathrm{HIi}}-$ see also Sec. VI.

Nevertheless, we can obtain a comprehensive and rather accurate approximation to the inflationary dynamics if we put $H \simeq H_{\mathrm{HI} 0}=\sqrt{V_{\mathrm{HI} 0}} / \sqrt{3} m_{\mathrm{P}}\left(H^{\prime}=0\right)$ and keep in Eq. (48) the most important terms in the expansion of $V_{\mathrm{HI}, f}$. In particular, we can easily verify that $V_{\mathrm{HI}, q}$ turns out to be proportional to $q$. As a consequence, Eq. (48) for $f=q$ is a second order linear homogeneous differential equation which admits an oscillatory solution with decreasing amplitude. Therefore, $q$ rapidly decreases to zero and so it does not influence the dynamics of the other fields. On the contrary, the e.o.m. of $s$ is non-homogenous as we can realize by inserting into Eq. (48) for $f=s$ the expression

$$
V_{\mathrm{HI}, s} \simeq V_{\mathrm{HIO}}\left(\frac{m_{+}^{2}}{m_{\mathrm{P}}^{2}} s-\frac{2 \kappa^{2} C_{1}+\left(\lambda-\mathrm{a} \kappa_{a}\right)^{2} C_{2}}{8\left(1-\mathrm{a}^{2}\right) \pi^{2} \sigma}\right),
$$

where the last two terms in the RHS of this equation have been derived by expanding the exact result for $s / \sigma \rightarrow 0$. Here

$$
C_{1}=\frac{N_{S}}{D_{S}} \text { and } C_{2}=\frac{\lambda^{2} N_{S}+\kappa_{a}^{2} N_{P}+2 \lambda \kappa_{a} N_{S P}}{\lambda^{2} D_{S}+\kappa_{a}^{2} D_{P}+2 \lambda \kappa_{a} D_{S P}}
$$

where we have used the abbreviations:

$$
\begin{aligned}
& N_{S[P]}=\frac{N_{+}^{2} U_{-}}{A_{-}^{2}}+\frac{N_{-}^{2} U_{+}}{A_{+}^{2}}-[+] \frac{N_{-} N_{+}\left(U_{-}+U_{+}\right)}{A_{+} A_{-}}, \\
& N_{S P}=\frac{N_{-}^{2} U_{+}}{A_{+}^{2}}-\frac{N_{+}^{2} U_{-}}{A_{-}^{2}}, D_{S P}=\frac{N_{-}^{2} U_{+}^{2}}{A_{+}^{2}}-\frac{N_{+}^{2} U_{-}^{2}}{A_{-}^{2}}, \\
& D_{S[P]}=\left(\frac{N_{+} U_{-}}{A_{-}}-[+] \frac{N_{-} U_{+}}{A_{+}}\right)^{2} .
\end{aligned}
$$

Since the general solution of the corresponding homogenous differential equation rapidly decreases to zero, as the field $q$, the solution of Eq. (48) with $f=s$, is dominated by the following particular solution

$$
s \simeq \frac{2 \kappa^{2} C_{1}+\left(\lambda-a \kappa_{a}\right)^{2} C_{2}}{8\left(1-a^{2}\right) \pi^{2} m_{+}^{2} \sigma} m_{\mathrm{P}}^{2},
$$

which minimizes $V_{\mathrm{HI}}$ in the $s$-direction, as can be seen from Eq. (51). In sharp contrast to the situation of Refs. [44 46], in our case $s$ turns out to be just mildly, and not drastically, reduced w.r.t. $\sigma$.

In the slow-roll approximation, which is determined by the condition

$$
\max \{\epsilon(\sigma),|\eta(\sigma)|\} \leq 1
$$

where

$$
\epsilon \simeq \frac{m_{\mathrm{P}}^{2}}{2}\left(\frac{V_{\mathrm{HI}, \sigma}}{V_{\mathrm{HI}}}\right)^{2} \text { and } \eta \simeq m_{\mathrm{P}}^{2} \frac{V_{\mathrm{HI}, \sigma \sigma}}{V_{\mathrm{HI}}},
$$

the e.o.m. of $\sigma$ in Eq. (48) takes the form $-3 H^{2} \sigma^{\prime} \simeq$ $V_{\mathrm{HI}, \sigma}$ or

$$
-\sigma^{\prime} \simeq m_{-}^{2} \sigma+\frac{m_{\mathrm{P}}^{2}\left(2 \kappa^{2}+\left(\lambda-\mathrm{a} \kappa_{a}\right)^{2}\right)}{8 \pi^{2}\left(1-\mathrm{a}^{2}\right) \sigma}+\frac{C_{3} \sigma^{3}}{m_{\mathrm{P}}^{2}\left(1-\mathrm{a}^{2}\right)^{2}},
$$

where - keeping only the most important terms in the expansion of the second term of the RHS of Eq. (18) we have

$$
\begin{aligned}
C_{3}= & \frac{1}{16\left(U_{-}-U_{+}\right)^{4}}\left(B_{1} D_{S}^{2}+B_{2} D_{P}^{2}\right. \\
& \left.+B_{3} D_{S P}^{2}+2 B_{4} D_{S P} D_{S}\right) .
\end{aligned}
$$

Solving Eq. (57) w.r.t. $\sigma$, we get

$$
\begin{aligned}
\sigma= & \frac{m_{\mathrm{P}}\left(1-\mathrm{a}^{2}\right)}{\sqrt{2 C_{3}}}\left[-m_{-}^{2}-D \tan (D N\right. \\
& \left.\left.-\arctan \frac{1}{D}\left(m_{-}^{2}+\frac{2 C_{3} \sigma_{\mathrm{HI}}^{2}}{m_{\mathrm{P}}^{2}\left(1-\mathrm{a}^{2}\right)^{2}}\right)\right)\right]^{1 / 2}
\end{aligned}
$$

with $\sigma_{\mathrm{HIi}}$ being the initial value of $\sigma$ at the beginning of FHI and

$$
D=\sqrt{C_{3}\left(2 \kappa^{2}+\left(\lambda-a \kappa_{a}\right)^{2}\right) / 2 \pi^{2}\left(1-a^{2}\right)^{3}-m_{-}^{4}} .
$$

The end of FHI takes place at $\sigma=\sigma_{\mathrm{f}}$, when the conditions of Eq. (10) or Eq. (55) are violated. For $\kappa \lesssim 0.005$, one can consider the former case only since the slow-roll conditions are violated infinitesimally close to the relevant critical point of the inflationary trajectory.

Soon afterwards, the inflaton system consisting of the two complex scalar fields $S$ and $(\delta \bar{\Phi}+\delta \Phi) / \sqrt{2}$ (where $\delta \bar{\Phi}=\bar{\Phi}-M$ and $\delta \Phi=\Phi-M)$ with mass $m_{1 \text { inf }}=\sqrt{2} \kappa M$ settles into a phase of damped oscillations about the SUSY vacuum and decays to MSSM degrees of freedom 
reheating the universe. The predominant decay channels of $S$ and $(\delta \bar{\Phi}+\delta \Phi) / \sqrt{2}$ are to fermionic and bosonic $\bar{Q}, Q$ respectively via tree-level couplings derived from the last term in the RHS of Eq. (11) with a common decay width

$$
\Gamma_{1}=\frac{1}{16 \pi} \lambda^{2} m_{\mathrm{inf}}^{2}
$$

The corresponding reheat temperature is [47] given by

$$
T_{1 \mathrm{rh}}=\left(\frac{72}{5 \pi^{2} g_{1 \mathrm{rh} *}}\right)^{1 / 4} \sqrt{\Gamma_{1} m_{\mathrm{P}}}
$$

where $g_{1 \mathrm{rh} *}=g_{*}\left(T_{1 \mathrm{rh}}\right)$ counts the effective number of relativistic degrees of freedom at temperature $T_{1 \mathrm{rh}}$. We find $g_{1 \mathrm{rh} *} \simeq 438.75\left[g_{1 \mathrm{rh} *} \simeq 513.75\right]$ for the MSSM spectrum plus right handed neutrinos and the particle content of the superfields $P, \bar{Q}, Q, \bar{D}_{\mathrm{a}}, D_{\mathrm{a}}$, and $H_{\mathrm{a}}$ for $n=5$ $[n=7]$.

For relatively large $\lambda^{\prime} \mathrm{s}(\simeq 0.05-0.1)$, we get $T_{1 \mathrm{rh}}>$ $V_{\mathrm{PQ} 0}^{1 / 4}$. As a consequence, after the end of FHI, we obtain matter domination (MD) for $T \geq T_{1 \text { rh }}$ and radiation domination $(\mathrm{RD})$ for $V_{\mathrm{PQ0}}^{1 / 4} \lesssim T \lesssim T_{1 \text { rh. }}$. During MD the evolution of $s$ can be found by solving its e.o.m. for $N>N_{\mathrm{HI}}$, i.e., inserting [45, 46, 48]

$$
H=H_{\mathrm{HIO}} e^{-3\left(N-N_{\mathrm{HI}}\right) / 2}, V=\frac{3 H^{2}}{4} s^{2}
$$

into Eq. (48). Taking also into account that, during the MD epoch, $R \propto \rho_{\text {osc }}^{-1 / 3}$ (where $\rho_{\text {osc }}$ is the energy density of the oscillating system), we can derive the value of $s$ at the beginning of PQPT:

$$
s_{\mathrm{PQi}} \simeq\left(\frac{\rho_{1 \mathrm{rh}}}{V_{\mathrm{HIO}}}\right)^{1 / 4} s_{\mathrm{HIf}} \text { with } \rho_{1 \mathrm{rh}}=\frac{\pi^{2}}{30} g_{1 \mathrm{rh} *} T_{1 \mathrm{rh}}^{4}
$$

being the radiation energy density at temperature $T_{1 \mathrm{rh}}$ and $s_{\text {HIf }}$ the value of $s$ at the end of FHI, which can be approximated by inserting $\sigma=\sigma_{\mathrm{f}}$ into Eq. (54). Note that during the subsequent $\mathrm{RD}$ era, $s$ remains frozen (see Ref. [41] and footnote 1 in Ref. 49]) and so the further reduction of $s$ relative to $\sigma$ can be evaded - cf. Ref. [44].

\section{THE STAGE OF PQPT}

For $T \lesssim V_{\mathrm{HI} 0}^{1 / 4}$, the cosmological dynamics is governed by the second term of $W$ in the RHS of Eq. (11). The SUGRA corrections can be safely ignored since $|P| \sim$ $f_{a} \ll m_{\mathrm{P}}$. The relevant F-term scalar potential is

$$
V_{\mathrm{PQF}}=\kappa_{a}^{2}\left|\bar{Q} Q-f_{a}^{2} / 4\right|^{2}+\kappa_{a}^{2}|P|^{2}\left(|\bar{Q}|^{2}+|Q|^{2}\right) .
$$

Along the F-flat direction in Eq. (11), $V_{\mathrm{PQF}}$ takes the constant value in Eq. (12), which breaks SUSY creating a mass splitting in the supermultiples $Q$ and $\bar{Q}$ and giving rise to one-loop radiative correction, $V_{\mathrm{PQc}}$, to the relevant potential - cf. Sec. III A 2 Indeed, the particle spectrum there includes a Dirac spinor with mass $\kappa_{a}|P|$ and 2 complex scalars with mass squared $\kappa_{a}^{2}\left(|P|^{2} \pm f_{a}^{2} / 4\right)$ and, therefore, $V_{\mathrm{PQc}}$ can be written [42] as follows:

$$
V_{\mathrm{PQc}}=\frac{\kappa_{a}^{2} V_{\mathrm{PQ} 0}}{32 \pi^{2}}\left(2 \ln \frac{\kappa_{a}^{2} A_{P}^{2} s^{2}}{\Lambda^{2}}+f_{\mathrm{rc}}\left(x_{s}\right)\right),
$$

where

$$
A_{P}=\frac{A_{-} N_{-}+A_{+} N_{+}}{A_{+} A_{-}\left(U_{-}-U_{+}\right)}
$$

with $x_{s}=A_{P} s / f_{a}$ and $P=A_{P} s / 2-$ see Eqs. (27), (39), and (40). When $|P|<f_{a} / 2$, one mass squared becomes negative and suggests a phase transition along the $|P|$ axis. Assuming gravity mediated soft SUSY breaking, the potential there has the form

$$
V_{\mathrm{PQ}}=V_{\mathrm{PQ} 0}+\frac{1}{2} m_{s}^{2} s^{2}-\sqrt{2 V_{\mathrm{PQ} 0}}\left|\mathrm{a}_{s}\right| s+V_{\mathrm{PQc}}
$$

for $s \geq f_{a} / A_{P}$. Here, $m_{s}$ is the soft SUSY breaking mass of $s$ and $\mathrm{a}_{s}$ is the soft SUSY breaking tadpole [39]. In Eq. (68), we take the phase of $\mathrm{a}_{s}$ to be $\arg \left(\mathrm{a}_{s}\right)=\pi$ which minimizes the $V_{\mathrm{PQ}}$ for given $s>0$. For reasonable values of the parameters involved, $V_{\mathrm{PQ}}$ does not give rise to inflation, mainly due to the contribution of $V_{\mathrm{PQc}}$ which spoils the $\eta$-criterion - and has not been taken into account in the analysis of Ref. [1]. This negative result remains even if we consider dissipative effects [50] due to the presence of the extra superpotential terms in Eq. (13).

Therefore, we are obliged to assume that after a negligible number of e-folds, the system consisting of the two complex scalar fields $P$ and $(\delta \bar{Q}+\delta Q) / \sqrt{2}$ (where $\delta \bar{Q}=$ $\bar{Q}-f_{a} / 2$ and $\left.\delta Q=Q-f_{a} / 2\right)$ with mass $m_{\mathrm{PQ}}=\kappa_{a} f_{a} / \sqrt{2}$ enters into an oscillatory phase about the PQ minimum and eventually decays, via the first non-renormalizable coupling in the RHS of Eq. (3), to Higgses and Higgsinos respectively with a common decay width [3]

$$
\Gamma_{2}=\frac{1}{2 \pi} \lambda_{\mu}^{2}\left(\frac{f_{a}}{2 m_{\mathrm{P}}}\right)^{2} m_{\mathrm{PQ}}
$$

The corresponding reheat temperature is [47] calculated by

$$
T_{2 \mathrm{rh}}=\left(\frac{72}{5 \pi^{2} g_{2 \mathrm{rh} *}}\right)^{1 / 4} \sqrt{\Gamma_{2} m_{\mathrm{P}}},
$$

where $g_{2 \mathrm{rh} *}=228.75$ is the energy density effective number of degrees of freedom for the MSSM spectrum.

\section{OBSERVATIONAL CONSTRAINTS}

Under the assumptions that (i) the curvature perturbation generated by $\sigma$ is solely responsible for the observed curvature perturbation and (ii) the violation of Eq. (10) occurs along the lines of Sec. IIB the parameters of our model can be restricted imposing the following requirements: 
a. According to the inflationary paradigm, the horizon and flatness problems of the standard Big Bang (SBB) can be successfully resolved provided that the number of e-foldings, $N_{\mathrm{HI} *}$, that the scale $k_{*}=$ $0.002 / \mathrm{Mpc}$ suffers during FHI takes a certain value which depends on the details of the cosmological scenario. Employing standard methods [20], we can easily derive the required $N_{\mathrm{HI} *}$ at $k_{*}$ :

$$
N_{\mathrm{HI} *} \simeq 23+\frac{2}{3} \ln \frac{V_{\mathrm{HI} 0}^{1 / 4}}{1 \mathrm{GeV}}-\frac{1}{3} \ln \frac{V_{\mathrm{PQ} 0}^{1 / 4}}{1 \mathrm{GeV}}+\frac{1}{3} \ln \frac{T_{1 \mathrm{rh}} T_{2 \mathrm{rh}}}{1 \mathrm{GeV}^{2}}
$$

consistently with the fact that $T_{1 \mathrm{rh}}>V_{\mathrm{PQ} 0}^{1 / 4}$ and, thus, we obtain $\mathrm{MD}$ followed by $\mathrm{RD}$ during the era between the end of FHI and the onset of PQPT. On the other hand, $N_{\mathrm{HI} *}$ can be found from

$$
N_{\mathrm{HI} *}=N_{\mathrm{HI}}-N_{*},
$$

where $N_{\mathrm{HI}}$ is the total number of e-foldings generated during FHI and $N_{*}$ is the number of e-foldings elapsed from the onset of FHI until the scale $k_{*}$ crosses outside the horizon of FHI and corresponds to the field value $\sigma_{*}$.

b. The power spectrum $P_{\mathcal{R}}$ of the curvature perturbation, which is generated during FHI and can be calculated at the pivot scale $k_{*}$ as a function of $\sigma_{*}$, is to be confronted with the WMAP7 data [17], i.e.

$$
P_{\mathcal{R}}^{1 / 2}=\left.\frac{1}{2 \sqrt{3} \pi m_{\mathrm{P}}^{3}} \frac{V_{\mathrm{HI}}^{3 / 2}}{\left|V_{\mathrm{HI}, \sigma}\right|}\right|_{\sigma=\sigma_{*}} \simeq 4.93 \times 10^{-5}
$$

c. The (scalar) spectral index $n_{\mathrm{s}}$, which is given by

$$
n_{\mathrm{s}}=1-6 \epsilon\left(\sigma_{*}\right)+2 \eta\left(\sigma_{*}\right)
$$

is to be consisted with the fitting of the WMAP7 data by the standard power-low $\Lambda \mathrm{CDM}$ :

$$
n_{\mathrm{s}}=0.963 \pm 0.028 \Rightarrow 0.935 \lesssim n_{\mathrm{s}} \lesssim 0.991
$$

at $95 \%$ confidence level (c.l.). Note, in passing, that the running of $n_{\mathrm{s}}$ and the scalar-to-tensor ratio turn out to be vanishingly small in our model as in any model of FHI.

d. In order for the PQPT to take place after a short temporary domination of $V_{\mathrm{PQ} 0}$, the value of $|P|$ at the onset of PQPT, $\left|P_{\mathrm{PQi}}\right|$, must be adequately larger than its critical value along the F-flat direction in Eq. (11). This entails

$$
\left|P_{\mathrm{PQi}}\right|>f_{a} / 2 \Rightarrow s_{\mathrm{PQi}}>f_{a} / A_{P}
$$

which implies mainly a lower bound on the parameter $\lambda$ through Eqs. (62) and (64). As we emphasize in Sec. IIA. this requirement is directly related to the reduction of the $\widetilde{G}$ yield at the onset of nucleosynthesis, $Y_{\widetilde{G}}$, to an acceptable level. Indeed, $Y_{\widetilde{G}}$ in the case that $V_{\mathrm{PQ} 0}$ does not dominate can be estimated [27] as

$$
Y_{1 \widetilde{G}} \simeq 1.9 \times 10^{-12}\left(T_{1 \mathrm{rh}} / 10^{10} \mathrm{GeV}\right)
$$

However, in the opposite case, if we take into account the entropy produced after PQPT during the subsequent reheating process - for computational details see Appendix $\mathrm{A}$ - we obtain

$$
Y_{2 \widetilde{G}} \simeq\left(\frac{\pi^{2}}{30} g_{1 \mathrm{rh} *}\right)^{1 / 4} \frac{T_{2 \mathrm{rh}}}{V_{\mathrm{PQ} 0}^{1 / 4}} Y_{1 \widetilde{G}}
$$

which is suppressed relative to $Y_{1 \widetilde{G}}$. In order to avoid spoiling the success of the SBB nucleosynthesis, an upper bound on $Y_{\widetilde{G}}$ is to be imposed depending on the $\widetilde{G}$ mass, $m_{\widetilde{G}}$, and the dominant $\widetilde{G}$ decay mode. For the conservative case that $\widetilde{G}$ decays with a tiny hadronic branching ratio, we have

$$
Y_{\widetilde{G}} \lesssim\left\{\begin{array} { l } 
{ 1 0 ^ { - 1 4 } } \\
{ 1 0 ^ { - 1 3 } } \\
{ 1 0 ^ { - 1 2 } }
\end{array} \text { for } m _ { \widetilde { G } } \simeq \left\{\begin{array}{l}
0.69 \mathrm{TeV} \\
10.6 \mathrm{TeV} \\
13.5 \mathrm{TeV}
\end{array}\right.\right.
$$

respectively. The bound above can be somehow relaxed in the case of a stable $\widetilde{G}$. However, it is achievable in our model, as we see below.

\section{NUMERICAL RESULTS}

As can be easily seen from the relevant expressions above, our cosmological set-up depends on the following parameters:

$$
\kappa, \kappa_{a}, \lambda, M, f_{a}, \lambda_{\mu}, n, a, b, c, d, e, f \text {, and } g .
$$

We fix $f_{a}=10^{12} \mathrm{GeV}$ and $\lambda_{\mu}=0.01$ so as to obtain $\mu \sim 1 \mathrm{TeV}$. We also set $n=5$. These three parameters are involved in the determination of $T_{1 \mathrm{rh}}$ and $T_{2 \mathrm{rh}}-$ via Eq. (62) and (70) respectively - and play no crucial role in the inflationary dynamics. We also choose $\kappa_{a}=0.01$ and $\lambda=0.1$. Variation of $\kappa_{a}$ leads to a variation of $s$ according to Eq. (54) without creating drastic changes in the inflationary predictions for $M, N_{\mathrm{HI} *}$, and $n_{\mathrm{s}}$. On the other hand, $\lambda$ controls crucially $s$ and $T_{1 \text { rh }}$ through Eqs. (54) and (64) and the upper bound on the condition of Eq. (10). Finally, we fix throughout our numerical computation $c=d=e=f=g=0.1$ since these parameters - contrary to a and $b$ - have a minor impact on the calculation of $m_{+}^{2}$ and $m_{-}^{2}$. As we show below, the selected values above give us a wide and natural allowed region of the remaining fundamental inflationary parameters $(\kappa, M, \mathrm{a}$, and $b)$. Besides the parameters above, in our computation, we use as input parameters the quantities $N_{*}$ and $f_{\mathrm{HIi}}=I$ with $f=\sigma, s$, and $q$. We set $I \simeq(1.5-4.5) \times 10^{17} \mathrm{GeV}$ so as to obtain $N_{\mathrm{HI}} \simeq 70-100$. We then restrict $M$ and $N_{*}$ so that Eqs. (71) and (733) are fulfilled. We check also if the violation of Eqs. (10a) and Eq. (10b) occurs according to the desired order so that our scenario is realized successfully. Using Eqs. (64) and (74), we can extract $s_{\mathrm{PQi}}$ and $n_{\mathrm{S}}$ and compare them with the requirements of Eqs. (76) and (75). For the solutions presented below, Eq. (76) is safely fulfilled. 


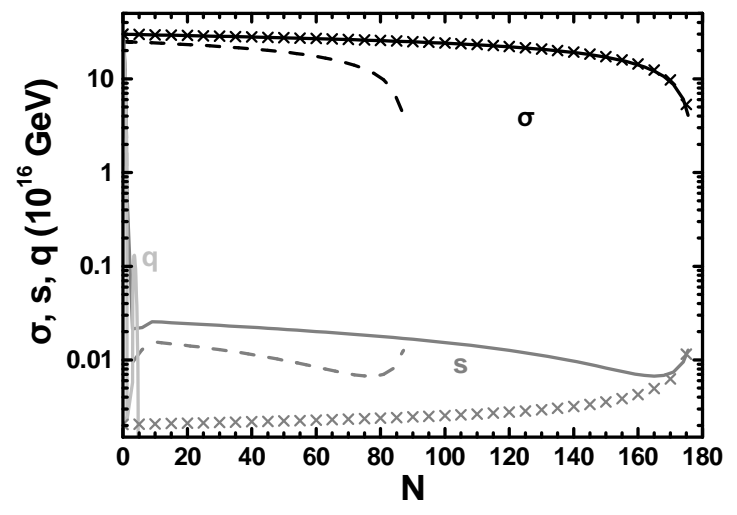

FIG. 1: The evolution of $\sigma$ (black lines), $s$ (gray lines), and $q$ (light gray lines) as evaluated via the solution of Eq. (48) as functions of $N$ for $\kappa=0.002, \kappa_{a}=-b=$ $0.01, \mathrm{a}=-0.0125, \lambda=c=d=e=f=g=0.1$, and $\sigma_{\mathrm{HIi}}=s_{\mathrm{HIi}}=q_{\mathrm{HIi}}=3 \times 10^{17} \mathrm{GeV}$ (solid lines) or $\sigma_{\mathrm{HIi}}=s_{\mathrm{HIi}}=q_{\mathrm{HIi}}=2.5 \times 10^{17} \mathrm{GeV}$ (dashed lines). Crosses are obtained by applying our analytical approach for $\sigma_{\mathrm{HIi}}=$ $s_{\mathrm{HIi}}=q_{\mathrm{HIi}}=3 \times 10^{17} \mathrm{GeV}$.

The selected $I$ is to be large enough so that $s$ reaches the attractor of Eq. (54). Under this assumption, our results are independent of the precise $I$, as can be clearly deduced from Fig. 1, where we plot $\sigma$ (black lines and crosses), $s$ (gray lines and crosses), and $q$ (light gray lines) as functions of $N$ for $\kappa=0.002, a=-0.0125, b=-0.01$, and $\sigma_{\mathrm{HIi}}=s_{\mathrm{HIi}}=q_{\mathrm{HIi}}=2.5 \times 10^{17} \mathrm{GeV}$ (dashed lines) or $\sigma_{\mathrm{HIi}}=s_{\mathrm{HIi}}=q_{\mathrm{HIi}}=3 \times 10^{17} \mathrm{GeV}$ (solid lines and crosses). The lines are drawn by solving numerically Eq. (48), whereas crosses are obtained by employing Eqs. (54) and (59). For both choices of $\sigma_{\mathrm{HIi}}=$ $s_{\mathrm{HIi}}=q_{\mathrm{HIi}}$ 's, we obtain $m_{-}^{2}=-0.0136, m_{+}^{2}=1.82$, $N_{\mathrm{HI} *}=52.5, M=2.86 \times 10^{16} \mathrm{GeV}, n_{\mathrm{s}}=0.963$, $\sigma_{\text {HIf }}=4.08 \times 10^{16} \mathrm{GeV}$, and $s_{\text {HIf }}=1.3 \times 10^{14} \mathrm{GeV}$ although in the first [second] case we obtain $N_{\mathrm{f}}=87.1$ $\left[N_{\mathrm{f}}=175.5\right]$, where $N_{\mathrm{f}}$ is the number of e-foldings elapsed from the commencement of FHI until Eq. (10 $)$ is violated. It is impressive that $M$ can take a value exactly equal to the SUSY GUT breaking scale, $M_{\mathrm{GUT}}=$ $2.86 \times 10^{16} \mathrm{GeV}$, contrary to all other realizations of the standard FHI - cf. Refs. [2, 19, 24, 36]. Despite the fact that $T_{1 \mathrm{rh}}=4.7 \times 10^{13} \mathrm{GeV}, s_{\mathrm{PQi}} / A_{P} f_{a} \simeq 23$ and, therefore, a second reheating process is possible which results to $T_{2 \mathrm{rh}}=3 \times 10^{4} \mathrm{GeV}$. It is worth emphasizing that $Y_{1 \widetilde{G}} \simeq 9 \times 10^{-9}$, whereas $Y_{2 \widetilde{G}} \simeq 1.9 \times 10^{-14}$ which is consistent with the constraint of Eq. (79). If we set $\lambda_{\mu}=0.05$, we take $\mu \simeq 5 \mathrm{TeV}, T_{2 \mathrm{rh}}=1.5 \times 10^{5} \mathrm{GeV}$, and $Y_{2 \widetilde{G}} \simeq 9.5 \times 10^{-14}$ which again falls into the ranges of Eq. (79). Finally, we observe that our analytical findings on $\sigma$ are very close to the numerical ones for all $N$ 's. On the contrary, analytical and numerical results on $s$ converge mainly for $N \simeq N_{\mathrm{f}}$. Let us clarify that the results presented in the following are derived exclusively by our

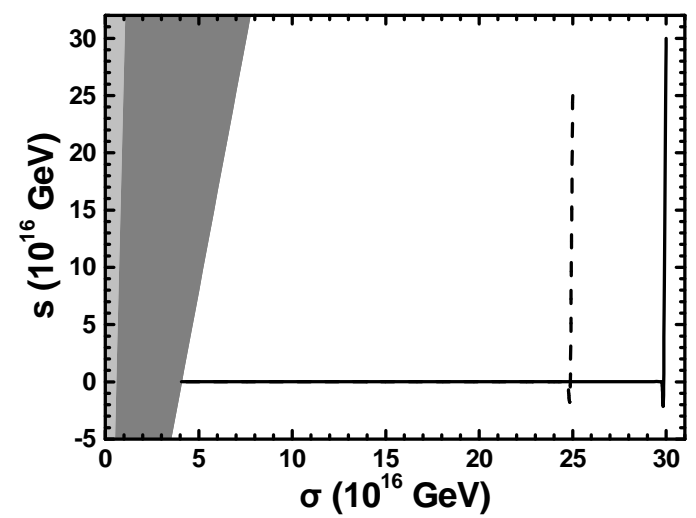

FIG. 2: The evolution of $\sigma$ and $s$ as evaluated via the solution of Eq. (48) in the $\sigma-s$ plane for $\kappa=0.002, \kappa_{a}=-b=$ $0.01, \mathrm{a}=-0.0125, \lambda=c=d=e=f=g=0.1$, and $\sigma_{\mathrm{HIi}}=s_{\mathrm{HIi}}=3 \times 10^{17} \mathrm{GeV}$ (solid line) or $\sigma_{\mathrm{HIi}}=s_{\mathrm{HIi}}=$ $2.5 \times 10^{17} \mathrm{GeV}$ (dashed line). The gray [light gray] region is excluded by Eq. (10) [Eq. (10b)].

numerical program.

As observed in Fig. 1, immediately after the onset of FHI, $q$ decreases sharply and so it does not influence the inflationary dynamics. The evolution of the other two fields $(\sigma$ and $s$ ) can be represented in the $\sigma-s$ plane as in Fig. 2 where we show the evolution of $\sigma$ and $s$ for the parameters used in Fig. [1 and taking $\sigma_{\mathrm{HIi}}=s_{\mathrm{HIi}}=$ $3 \times 10^{17} \mathrm{GeV}$ (solid line) or $\sigma_{\mathrm{HIi}}=s_{\mathrm{HIi}}=2.5 \times 10^{17} \mathrm{GeV}$ (dashed line). There, we draw also the gray [light gray] region which is excluded by Eq. (10a) [Eq. (10b)]. We remark that Eq. (10a) is violated earlier and so the preferred hierarchy in the domination of $V_{\mathrm{HI} 0}$ or $V_{\mathrm{PQ} 0}$ in our cosmological proposal is valid - see Sec. IIA

The importance of the coefficient a in reducing $n_{\mathrm{S}}$ can be easily concluded from Fig. 3(a), where we depict $n_{\mathrm{s}}$ versus a for $b=-0.01$ and various $\kappa$ 's indicated in the graph. Increasing the absolute value of a, $\left|m_{-}^{2}\right|$ increases too and so $n_{\mathrm{s}}$ decreases and becomes consistent with the observationally favored range of Eq. (75). On the other hand, the same variation of a leads to a reduction of $M$ which lies around its SUSY GUT value, $M_{\mathrm{GUT}}$, as can be concluded from Fig. 3(b).

Confronting FHI with all the constraints of Sec. $\mathrm{V}$, we can delineate the allowed (lightly gray shaded) region in the $\kappa-\mathrm{a}[\kappa-M]$ plane as in Fig. 4(a) [Fig. 4(b)], where we take $b=-0.01$ and show the adopted conventions for the various lines. In particular, the gray dashed [dotted] lines correspond to $n_{\mathrm{s}}=0.991\left[n_{\mathrm{s}}=0.935\right]$, whereas the gray solid lines are obtained by fixing $n_{\mathrm{s}}=0.963$ - see Eq. (75). The black solid lines correspond to $M=M_{\mathrm{GUT}}$, whereas beyond the black dashed lines the preferred hierarchy in the violation of Eqs. (10a) and (10p) fails. We observe that the latter requirement holds only for $\kappa \lesssim \lambda$. Along the gray solid line $\left(n_{\mathrm{s}}=0.963\right)$, we obtain $2.7 \times 10^{13} \lesssim T_{1 \mathrm{rh}} / \mathrm{GeV} \lesssim 1.5 \times 10^{14}$ and 


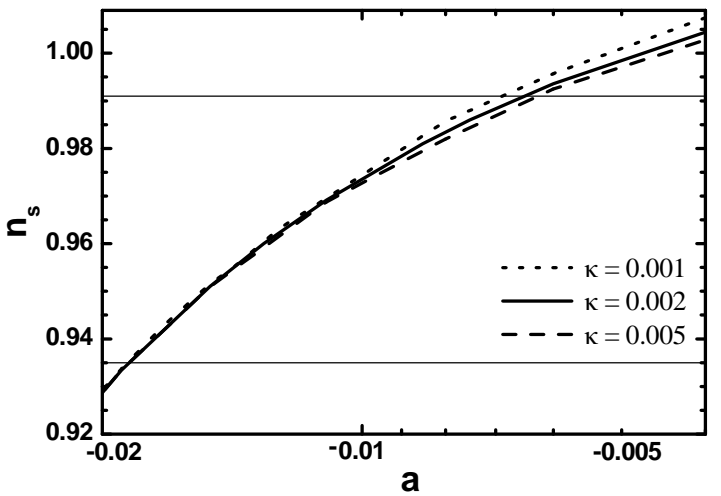

(a)

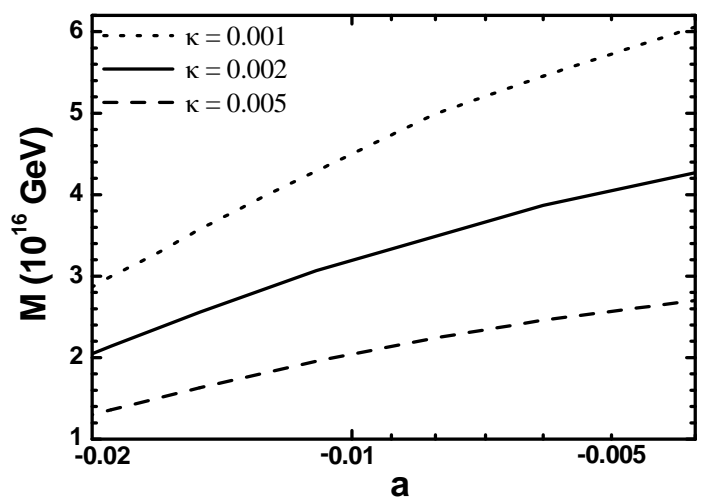

(b)

FIG. 3: The allowed by Eqs. (71) and (73) values of $n_{\mathrm{s}}(\mathrm{a})$ and $M(\mathrm{~b})$ versus a for $\kappa_{a}=-b=0.01, \lambda=c=d=e=f=g=0.1$, and several $\kappa$ 's indicated in the graphs. The region of Eq. (75) is also limited by thin lines in (a).

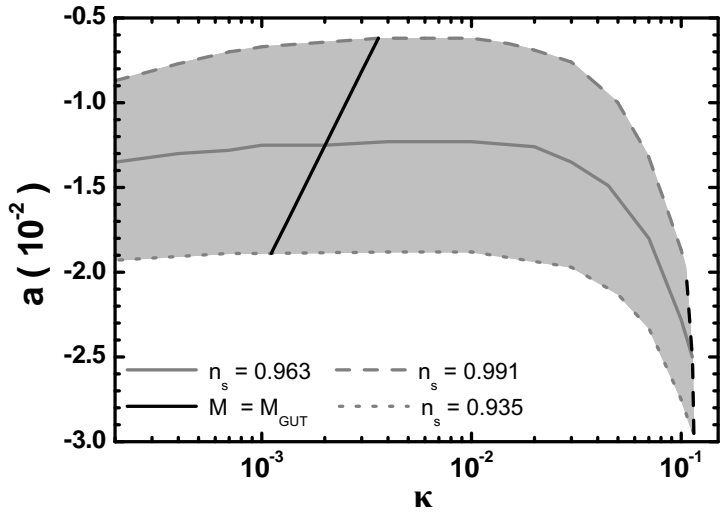

(a)

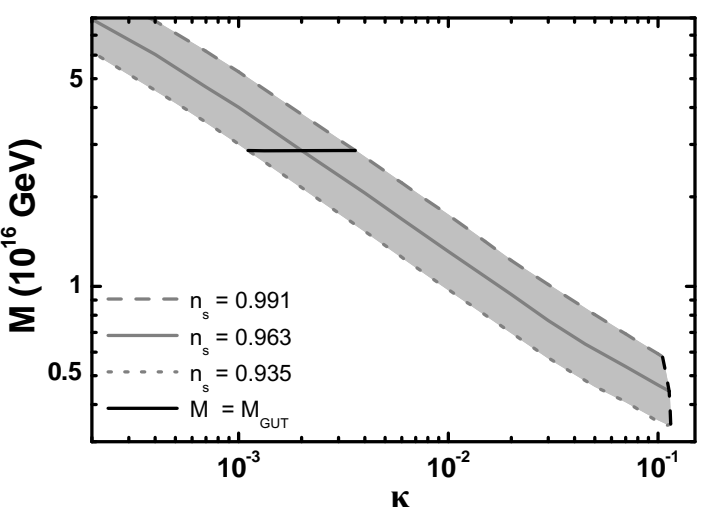

(b)

FIG. 4: Allowed (lightly gray shaded) regions in the (a) $\kappa-$ a and (b) $\kappa-M$ plane for $\kappa_{a}=-b=0.01$ and $\lambda=c=d=e=$ $f=g=0.1$. The gray dashed [dotted] lines correspond to the upper [lower] bound on $n_{\mathrm{s}}$ in Eq. (75), whereas the gray solid lines have been obtained by fixing $n_{\mathrm{s}}$ to its central value in Eq. (75). Along the black solid lines, we fix $M=M_{\mathrm{GuT}}$, whereas beyond the black dashed lines the preferred hierarchy in the violation of Eqs. (10) and (10) is broken. In the allowed regions, Eqs. (71) and (73) are also fulfilled.

$1.9 \times 10^{-14} \lesssim Y_{2 \widetilde{G}} \lesssim 10^{-13}$ for $2 \times 10^{-4} \lesssim \kappa \lesssim 0.113$ Note that $T_{2 \mathrm{rh}}=3 \times 10^{4} \mathrm{GeV}$ remains fixed since $\lambda_{\mu}$ and $f_{a}$ are also fixed throughout our computation.

One of the outstanding features of our proposal is that the reduction of $n_{\mathrm{s}}$ can be attained without disturbing the monotonicity of the potential, contrary to other similar suggestions - see Refs. [20, 23, 24]. This fact can be highlighted in Fig. 5, where we present the variation of the inflationary potential $V_{\mathrm{HI}}$ as a function of the inflaton field, $\sigma$, for the values of the parameters corresponding to the three intersections of the solid black line with the gray lines in Fig. 4(a) and (b). Namely, we take $M=M_{\mathrm{GUT}}, b=-0.01$, and $\kappa=0.0036, \mathrm{a}=-0.0062$, and $\sigma_{\mathrm{HIi}}=s_{\mathrm{HIi}}=q_{\mathrm{HIi}}=3 \times 10^{17} \mathrm{GeV}\left(n_{\mathrm{s}}=0.991\right.$, dashed line) or $\kappa=0.002, \mathrm{a}=-0.0125$, and $\sigma_{\mathrm{HIi}}=$ $s_{\mathrm{HIi}}=q_{\mathrm{HIi}}=2.5 \times 10^{17} \mathrm{GeV}\left(n_{\mathrm{s}}=0.963\right.$, solid line $)$ or $\kappa=0.00111, \mathrm{a}=-0.0189$, and $\sigma_{\mathrm{HIi}}=s_{\mathrm{HIi}}=q_{\mathrm{HIi}}=$
$2 \times 10^{17} \mathrm{GeV}\left(n_{\mathrm{s}}=0.935\right.$, dotted line). The values corresponding to $\sigma_{*}$ and $\sigma_{\mathrm{f}}$ are also designed. We observe that for large values of $\sigma, V_{\mathrm{HI}}$ develops an oscillatory behavior due to the initial oscillations of $s$ and $q-$ see Fig. 1. However, $V_{\mathrm{HI}}$, for lower $\sigma$ 's, remains monotonic and, therefore, no complications arise in the realization of the inflationary dynamics.

Letting $b$ vary for a number of fixed values of $a$ and for the most exciting case with $M=M_{\mathrm{GUT}}$, we can depict the values allowed by all the constraints of Sec. $\mathrm{V}$ in the $\kappa-b$ plane - see Fig. 6. The various lines terminate at low [high] $\kappa$ 's due to the saturation of Eq. (75) from below [above]. The central $n_{\mathrm{s}}$ is obtained at $\kappa \simeq 0.002$. We readily conclude that the allowed a's and $b$ 's for $M=$ $M_{\mathrm{GUT}}$ and fixed $n_{\mathrm{s}}$ are almost $\kappa$-independent. This is because, for fixed $n_{\mathrm{s}}, m_{-}^{2}$ is fixed too. In particular, for $n_{\mathrm{s}}=0.963$, we have $m_{-}^{2}=-0.0136$, whereas, for $n_{\mathrm{s}}=$ 


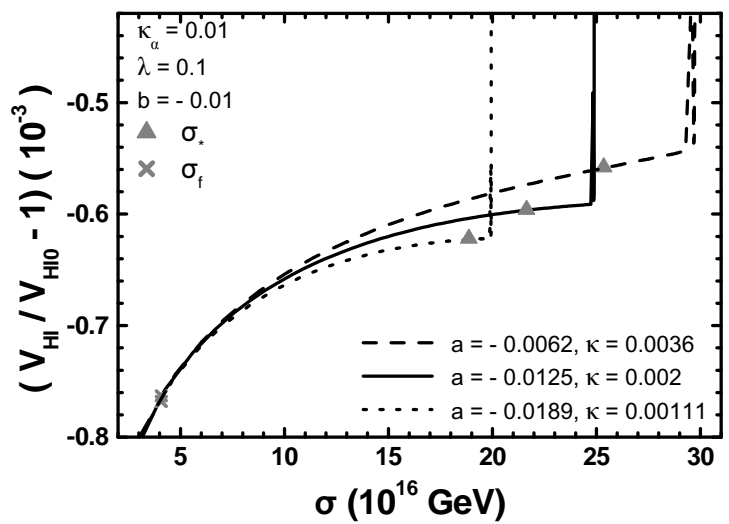

FIG. 5: The variation of the inflationary potential $V_{\mathrm{HI}}$ as a function of $\sigma$ for $M=M_{\mathrm{GUT}}, \kappa_{a}=-b=0.01, \lambda=c=d=$ $e=f=g=0.1$, and $\kappa=0.0036, \mathrm{a}=-0.0062$, and $\sigma_{\mathrm{HIi}}=$ $s_{\mathrm{HIi}}=q_{\mathrm{HIi}}=3 \times 10^{17} \mathrm{GeV}\left(n_{\mathrm{s}}=0.991\right.$, dashed line $)$ or $\kappa=$ $0.002, \mathrm{a}=-0.0125$, and $\sigma_{\mathrm{HIi}}=s_{\mathrm{HIi}}=q_{\mathrm{HIi}}=2.5 \times 10^{17} \mathrm{GeV}$ $\left(n_{\mathrm{s}}=0.963\right.$, solid line) or $\kappa=0.00111, \mathrm{a}=-0.0189$, and $\sigma_{\mathrm{HIi}}=s_{\mathrm{HIi}}=q_{\mathrm{HIi}}=2 \times 10^{17} \mathrm{GeV}\left(n_{\mathrm{s}}=0.935\right.$, dotted line $)$. The values corresponding to $\sigma_{*}$ and $\sigma_{\mathrm{f}}$ are also depicted.

$0.935\left[n_{\mathrm{s}}=0.991\right]$, we have $m_{-}^{2} \simeq-0.02\left[m_{-}^{2} \simeq-0.008\right]$ and $\kappa \simeq 0.0036[\kappa \simeq 0.0011]$. In all cases, $m_{+}^{2} \simeq 1.82$. For this reason, we can present in Fig. 7 the allowed values by Eqs. (71), (73), and (75) in the $a-b$ plane for $M \simeq M_{\mathrm{GUT}}$ and $\kappa \simeq 0.002\left(n_{\mathrm{s}}=0.963\right.$, solid lines $)$, $\kappa \simeq 0.0036\left(n_{\mathrm{s}}=0.991\right.$, dashed lines $)$ or $\kappa \simeq 0.0011$ ( $n_{\mathrm{s}}=0.935$, dotted lines). We observe that our scenario can be realized for both signs of a and $b$, contrary to the cases studied in Refs. [20, 23, 24] where negative $b$ 's are necessitated. Moreover, compared to the latter cases, larger $|b|$ 's (of the order of 0.1 ) are here permitted.

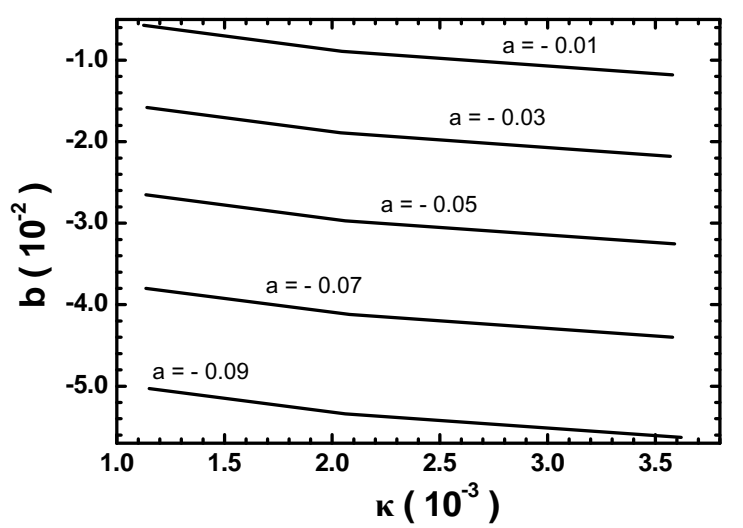

FIG. 6: Allowed values by Eqs. (71), (73), and (75) in the $\kappa-b$ plane for $\kappa_{a}=0.01, \lambda=c=d=e=f=g=0.1, M=$ $M_{\mathrm{GUT}}$, and various a's indicated on the curves.
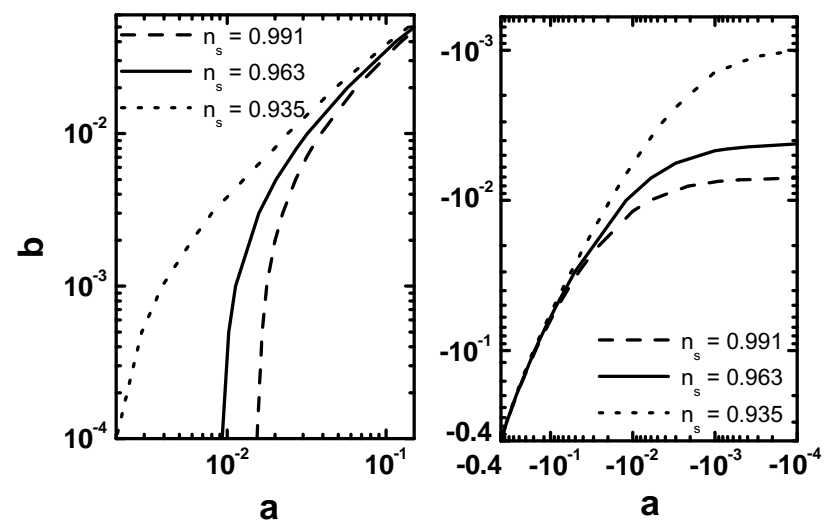

FIG. 7: Allowed values by Eqs. (71), (73), and (75) in the $a-b$ plane for $\kappa_{a}=0.01, \lambda=c=d=e=f=g=0.1, \quad M \simeq$ $M_{\mathrm{GUT}}$, and $\kappa \simeq 0.002$ (solid lines), $\kappa \simeq 0.0036$ (dashed lines) or $\kappa \simeq 0.0011$ (dotted lines).

\section{CONCLUSIONS}

We investigated a cosmological scenario according to which a SUSY GUT scale FHI is followed by a PQPT which resolves the strong $\mathrm{CP}$ and the $\mu$ problems of MSSM. The PQPT is tied to renormalizable superpotential terms and the possible catastrophic production of domain walls can be eluded by the introduction of extra matter superfields which can be chosen so that the MSSM gauge coupling constant unification is not disturbed. The inflaton-like field, associated with PQPT, plays a crucial role in the construction of the Kähler potential which is expanded up to fourth order in powers of the various fields. The FHI reproduces the current data on $P_{\mathcal{R}}$ and $n_{\mathrm{s}}$ within the power-law $\Lambda \mathrm{CDM}$ cosmological model and generates the number of e-folds required from the resolution of the horizon and flatness problems of the SBB. The dynamics of FHI is investigated both numerically and analytically and the results are compared with each other. Fixing $n_{\mathrm{s}}$ to its central value and $M$ to the SUSY GUT scale, we concluded that $\kappa=0.002$ with the remaining parameters taking more or less natural values, $\pm(0.01-0.1)$. It is gratifying that our model supports a second stage of reheating after PQPT, which dilutes sufficiently the $\widetilde{G}$ abundance so as to become observationally safe for $\widetilde{G}$ masses even lower than $10 \mathrm{TeV}$.

\section{Acknowledgments}

We would like to thank I. Moss, R.O. Ramos, V.N. Şenoğuz, and Q. Shafi for useful discussions. This work was supported by the European Union under the Marie Curie Initial Training Network "UNILHC" PITN-GA2009-237920 and the Marie Curie Research Training Network "UniverseNet" MRTN-CT-2006-035863. 


\section{Appendix A: Reheating Processes and Gravitino Constraint}

In this Appendix, we present a numerical description of the post-inflationary evolution of the various energy densities in our set-up paying special attention to the dilution of the $\widetilde{G}$ yield, $Y_{\widetilde{G}}$.

The energy density, $\rho_{1}\left[\rho_{2}\right]$, of the oscillatory system which reheats the universe at the temperature $T_{1 \mathrm{rh}}\left[T_{2 \mathrm{rh}}\right]$, the energy density of produced radiation, $\rho_{\mathrm{R}}$, and the

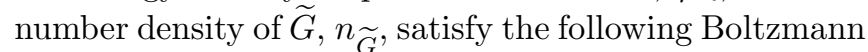
equations - cf. Refs. [23, 27]:

$$
\begin{aligned}
& \dot{\rho}_{1}+3 H \rho_{1}+\Gamma_{1} \rho_{1}=0, \\
& \dot{\rho}_{2}+3 H \rho_{2}+\Gamma_{2} \rho_{2}=0, \\
& \dot{\rho}_{\mathrm{R}}+4 H \rho_{\mathrm{R}}-\Gamma_{1} \rho_{1}-\Gamma_{2} \rho_{2}=0, \\
& \dot{n}_{\widetilde{G}}+3 H n_{\widetilde{G}}-C_{\widetilde{G}}\left(n^{\mathrm{eq}}\right)^{2}=0 .
\end{aligned}
$$

Here $n^{\text {eq }}=\zeta(3) T^{3} / \pi^{2}$ is the equilibrium number density of the bosonic relativistic species, $C_{\widetilde{G}}$ is a collision term for $\widetilde{G}$ production which, in the limit of the massless gauginos, turns out to be [27, 51]

$$
C_{\widetilde{G}}=\frac{3 \pi}{16 \zeta(3) m_{\mathrm{P}}^{2}} \sum_{i=1}^{3} c_{i} g_{i}^{2} \ln \left(\frac{k_{i}}{g_{i}}\right),
$$

where $g_{i}$ (with $i=1,2,3$ ) are the gauge coupling constants of MSSM, $\left(k_{i}\right)=(1.634,1.312,1.271)$, and $\left(c_{i}\right)=$ $(33 / 5,27,72)$. Also the Hubble expansion parameter, $H$, during this period is given by

$$
H=\frac{1}{\sqrt{3} m_{\mathrm{P}}}\left(m_{\widetilde{G}} n_{\widetilde{G}}+\rho_{1}+\rho_{2}+\rho_{\mathrm{R}}\right)^{1 / 2} .
$$

Clearly, in the limit of massless MSSM gauginos, the $n_{\widetilde{G}}$ computation is $m_{\widetilde{G}}$-independent. The temperature, $T$, and the entropy density, s (not to be confused with the field $s$ ), can be found using the relations

$$
\rho_{\mathrm{R}}=\frac{\pi^{2}}{30} g_{*} T^{4} \text { and } \mathrm{s}=\frac{2 \pi^{2}}{45} g_{*} T^{3},
$$

where $g_{*}(T)=g_{1 \mathrm{rh} *}\left[g_{*}(T)=g_{2 \mathrm{rh} *}\right]$ for $T \geq T_{\mathrm{PQ}}$ $\left[T<T_{\mathrm{PQ}}\right]$ with $T_{\mathrm{PQ}}$ being defined as the solution of the equation $\rho_{\mathrm{R}}\left(T_{\mathrm{PQ}}\right)=V_{\mathrm{PQ} 0}$ and can be found numerically.

The numerical integration of Eqs. (A1)-(A4) is facilitated by absorbing the dilution terms. To this end, we find it convenient to define [47] the following dimensionless variables

$$
f_{1}=\rho_{1} R^{3}, f_{2}=\rho_{2} R^{3}, f_{\mathrm{R}}=\rho_{\mathrm{R}} R^{4}, \text { and } f_{\widetilde{G}}=n_{\widetilde{G}} R^{3} .
$$

Converting the time derivatives to derivatives w.r.t $\bar{N}=$ $\ln \left(R / R_{\mathrm{HIf}}\right)$ with $R_{\mathrm{HIf}}$ being the value of the scale factor at the end of FHI (the value of $R_{\mathrm{HIf}}$ turns out to be numerically irrelevant), Eqs. (A1)-(A4) become

$$
H f_{1}^{\prime}=-\Gamma_{1} f_{1}
$$

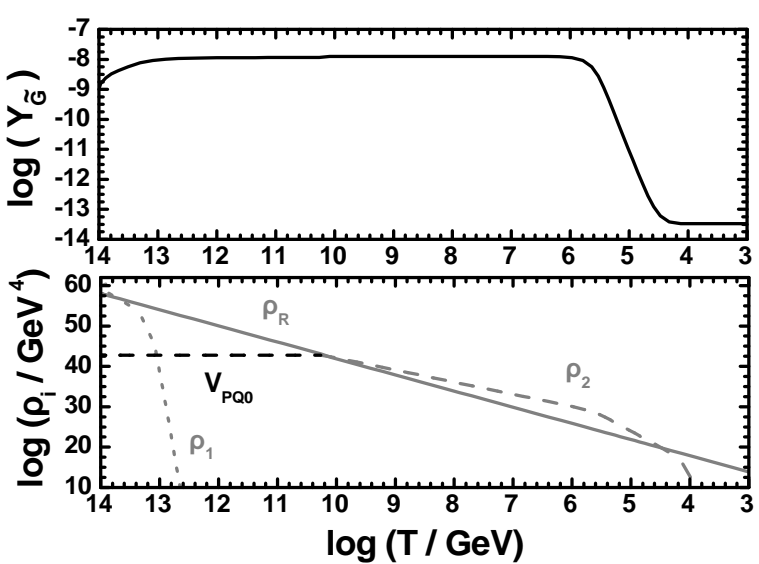

FIG. 8: The evolution of the quantities $\log \rho_{i}$ with $i=1$ (gray dotted line), $i=2$ (gray dashed line), $i=\mathrm{R}$ (gray line), $\log V_{\mathrm{PQ} 0}$ (black dashed line), and $\log Y_{\widetilde{G}}$ (black solid line) as functions of $\log T$ for $\kappa=0.002, \kappa_{a}=-b=0.01, \mathrm{a}=$ $-0.0125, \lambda=c=d=e=f=g=0.1, M=M_{\mathrm{GUT}}, f_{a}=$ $10^{12} \mathrm{GeV}$, and $\lambda_{\mu}=0.01$

$$
\begin{aligned}
H f_{2}^{\prime} & =-\Gamma_{2} f_{2}, \\
H f_{\mathrm{R}}^{\prime} & =\Gamma_{1} f_{1} R+\Gamma_{2} f_{2} R, \\
H f_{\widetilde{G}}^{\prime} & =C_{\widetilde{G}}\left(n^{\mathrm{eq}}\right)^{2} R^{3},
\end{aligned}
$$

where the differentiation w.r.t. $\bar{N}$ and $N$ - see Sec. IIIB - coincides. Also $H$ and $T$ can be expressed in terms of the variables in Eq. (A8) as

$$
H=\frac{\sqrt{m_{\widetilde{G}} f_{\widetilde{G}}+f_{1}+f_{2}+f_{\mathrm{R}} / R}}{\sqrt{3 R^{3}} m_{\mathrm{P}}} \text { and } T=\sqrt[4]{\frac{30 f_{\mathrm{R}}}{\pi^{2} g_{*} R^{4}}} .
$$

The system of Eqs. (A99) A12 can be solved, imposing the following initial conditions (the quantities below are considered functions of the independent variable $\bar{N}$ ):

$\rho_{1}(0)=V_{\mathrm{HI} 0}, \rho_{\mathrm{R}}(0)=n_{\widetilde{G}}(0)=0$, and $\rho_{2}\left(\bar{N}_{\mathrm{PQ}}\right)=V_{\mathrm{PQ} 0}$,

where $\bar{N}_{\mathrm{PQ}}$ is the value of $\bar{N}$ corresponding to the temperature $T_{\mathrm{PQ}}$. Needless to say that we set $\rho_{2}(\bar{N})=0$ for $\bar{N}<\bar{N}_{\mathrm{PQ}}$.

In Fig. 8, we illustrate the cosmological evolution of the quantities $\log \rho_{i}$ with $i=1$ (dotted gray line), $i=2$ (dashed gray line), and $i=\mathrm{R}$ (gray line), $\log V_{\mathrm{PQ} 0}$ (black dashed line), and $\log Y_{\widetilde{G}}$ (black solid line) as functions of $\log T$ for the values of the parameters adopted in Fig. 11 In particular, the parameters which determine the evolution of the various quantities during the post-inflationary period are $\kappa, \lambda, M, \kappa_{a}, f_{a}$, and $\lambda_{\mu}$. We take $\kappa=0.002, \lambda=0.1, M=M_{\mathrm{GUT}}, \kappa_{a}=0.01, f_{a}=$ $10^{12} \mathrm{GeV}$, and $\lambda_{\mu}=0.01$. From Fig. 8 , we observe that FHI is followed successively by the following four epochs: (i) a MD era, due to the oscillating and decaying inflaton system, which lasts until $T \simeq T_{1 \text { rh }}$ given by Eq. (62), (ii) a $\mathrm{RD}$ epoch, which terminates at $T_{\mathrm{PQ}}$, (iii) a MD era cre- 
ated by the oscillations of the PQ-system, which is completed at $T \simeq T_{2 \text { rh }}$ given by Eq. (70), and (iv) a RD epoch after which the universe enters the SBB phase. The completion of the two reheating processes corresponds to the two intersections of $\rho_{1}$ and $\rho_{2}$ with $\rho_{\mathrm{R}}$ in Fig. 8. Here we omit for simplicity any possible instantaneous domination of $V_{\mathrm{PQ} 0}$ over the various energy densities since, as we mention in Sec. IV] $V_{\mathrm{PQ}}$ does not support any significant period of inflation. In Fig. 8, we also see that the $\widetilde{G}$ yield, $Y_{\widetilde{G}}=n_{\widetilde{G}} / \mathrm{s}$, takes the value $Y_{1 \widetilde{G}} \simeq 1.1 \times 10^{-8}$ - see Eq. (77) - for $T \simeq T_{1 \mathrm{rh}}$. However, due to the entropy released during the out-of-equilibrium decay of the PQ system, $Y_{\widetilde{G}}$ decreases sharply to $Y_{2 \widetilde{G}} \simeq 3.3 \times 10^{-14}$ - see Eq. (78). This result can be understood from the following relation

$$
Y_{2 \widetilde{G}}=Y_{1 \widetilde{G}} \frac{\mathrm{s}\left(T_{\mathrm{PQ}}\right)}{\mathrm{s}\left(T_{2 \mathrm{rh}}\right)}\left(\frac{R_{\mathrm{PQ}}}{R_{2 \mathrm{rh}}}\right)^{3},
$$

where $R_{\mathrm{PQ}}\left[R_{2 \mathrm{rh}}\right]$ is the value of the scale factor corresponding to $T_{\mathrm{PQ}}\left[T_{2 \mathrm{rh}}\right]$. Taking into account Eq. (A7) and the fact that, during this $\mathrm{MD}$ era, $R \propto \rho_{\mathrm{osc}}^{-1 / 3}-$ recall that $\rho_{\text {osc }}$ is the energy density of the oscillating system - we arrive at Eq. (78). We verify that the analytical results agree remarkably with the numerical ones.

Therefore, we can easily appreciate the importance of PQPT, as realized in our scenario, in lowering $Y_{\widetilde{G}}$ to a value compatible with the observational data [27].
[1] E.J. Copeland, A.R. Liddle, D.H. Lyth, E.D. Stewart, and D. Wands, Phys. Rev. D 49, 6410 (1994).

[2] G.R. Dvali, Q. Shafi, and R.K. Schaefer, Phys. Rev. Lett. 73, 1886 (1994); G. Lazarides, R.K. Schaefer, and Q. Shafi, Phys. Rev. D 56, 1324 (1997).

[3] G. Lazarides, Lect. Notes Phys. 592, 351 (2002), arXiv:hep-ph/0111328; J. Phys. Conf. Ser. 53, 528 (2006), arXiv:hep-ph/0607032

[4] G.R. Dvali, G. Lazarides, and Q. Shafi, Phys. Lett. B 424, 259 (1998).

[5] G. Lazarides and Q. Shafi, Phys. Rev. D 58, 071702 (1998).

[6] R. Peccei and H. Quinn, Phys. Rev. Lett. 38, 1440 (1977); S. Weinberg, ibid. 40, 223 (1978); F. Wilczek, ibid. 40, 279 (1978).

[7] G. Lazarides, R.K. Schaefer, and Q. Shafi, Phys. Rev. D 56, 1324 (1997).

[8] R. Jeannerot, S. Khalil, G. Lazarides, and Q. Shafi, J. High Energy Phys. 10, 012 (2000).

[9] L. Dolan and R. Jackiw, Phys. Rev. D 9, 3320 (1974); S. Weinberg, ibid. 9, 3357 (1974).

[10] K. Dimopoulos, G. Lazarides, D. Lyth, and R. Ruiz de Austri, J. High Energy Phys. 05, 057 (2003).

[11] D. Seckel and M.S. Turner, Phys. Rev. D 32, 3178 (1985); M.S. Turner and F. Wilczek, Phys. Rev. Lett. 66, 5 (1991); M. Kawasaki and K. Nakayama, Phys. Rev. D 77, 123524 (2008).

[12] D.H. Lyth and E.D. Stewart, Phys. Rev. D 46 (1992) 532.

[13] J.E. Kim, Phys. Lett. B 136, 378 (1984); T. Goto and M. Yamaguchi, ibid. 276, 103 (1992).

[14] P. Sikivie, Phys. Rev. Lett. 48, 1156 (1982).

[15] G. Lazarides and Q. Shafi, Phys. Lett. B 115, 21 (1982); H. Georgi and M.B. Wise, ibid. 116, 123 (1982).

[16] G. Lazarides and Q. Shafi, Phys. Lett. B 489, 194 (2000).

[17] E. Komatsu et al. [WMAP Collaboration], arXiv:1001. 4538 [astro-ph].

[18] R.A. Battye, B. Garbrecht, and A. Moss, J. Cosmol. Astropart. Phys. 09, 007 (2006).

[19] G. Lazarides and C. Pallis, Phys. Lett. B 651, 216 (2007); G. Lazarides, Int. J. Mod. Phys. A 22, 5747 (2007); C. Pallis, AIP Conf. Proc. 1122, 368 (2009).

[20] C. Pallis, "High Energy Physics Research Advances", edited by T.P. Harrison and R.N. Gonzales (Nova Sci- ence Publishers Inc., New York, 2008), arXiv:0710.3074 [hep-ph].

[21] G. Lazarides and A. Vamvasakis, Phys. Rev. D 76, 083507 (2007); G. Lazarides and A. Vamvasakis, ibid. 76, 123514 (2007); G. Lazarides, I.N.R. Peddie, and A. Vamvasakis, ibid. 78, 043518 (2008); G. Lazarides, arXiv:1006.3636 [hep-ph].

[22] C. Pallis, J. Cosmol. Astropart. Phys. 04, 024 (2009); M. Ur Rehman, Q. Shafi, and J.R. Wickman, Phys. Lett. B 683, 191 (2010); M. Ur Rehman, Q. Shafi, and J.R. Wickman, ibid. 688, 75 (2010).

[23] B. Garbrecht, C. Pallis, and A. Pilaftsis, J. High Energy Phys. 12, 038 (2006).

[24] M. Bastero-Gil, S.F. King, and Q. Shafi, Phys. Lett. B 651, 345 (2007); M. ur Rehman, V.N. Şenoğuz, and Q. Shafi, Phys. Rev. D 75, 043522 (2007).

[25] L. Boubekeur and D. Lyth, J. Cosmol. Astropart. Phys. 07, 010 (2005).

[26] M.Yu. Khlopov and A.D. Linde, Phys. Lett. B 138, 265 (1984); J. Ellis, J.E. Kim, and D.V. Nanopoulos, ibid. 145, 181 (1984); I.V. Falomkin et al., Sov. J. Nucl. Phys. 39, 626 (1984); J.R. Ellis, D.V. Nanopoulos and S. Sarkar, Nucl. Phys. B259, 175 (1985); J.R. Ellis et al., ibid. B373, 399 (1992).

[27] M. Kawasaki, K. Kohri, and T. Moroi, Phys. Lett. B 625, 7 (2005); Phys. Rev. D 71, 083502 (2005); R.H. Cyburt et al., ibid. 67, 103521 (2003); J.R. Ellis, K.A. Olive, and E. Vangioni, Phys. Lett. B 619, 30 (2005).

[28] T.W.B. Kibble, J. Phys. A 9, 1387 (1976).

[29] M. Fukugita and T. Yanagida, Phys. Lett. B 174, 45 (1986).

[30] G. Lazarides and Q. Shafi, Phys. Lett. B 258, 305 (1991); G. Lazarides, arXiv:hep-ph/9905450.

[31] G. Lazarides, R. Ruiz de Austri, and R. Trotta, Phys. Rev. D 70, 123527 (2004).

[32] J. Rocher and M. Sakellariadou, J. Cosmol. Astropart. Phys. 03, 004 (2005); R. Jeannerot and M. Postma, J. High Energy Phys. 05, 071 (2005).

[33] G. Lazarides, C. Panagiotakopoulos, and Q. Shafi, Phys. Rev. Lett. 56, 432 (1986).

[34] N. Ganoulis, G. Lazarides, and Q. Shafi, Nucl. Phys. B323, 374 (1989); G. Lazarides and Q. Shafi, ibid. B329, 182 (1990).

[35] J. Kim, Phys. Rep. 150, 1 (1987). 
[36] C. Panagiotakopoulos and N. Tetradis, Phys. Rev. D 59, 083502 (1999).

[37] B. Kyae, arXiv:0910.4092 [hep-ph].

[38] See e.g. M. Peskin, arXiv:0801.1928 [hep-ph].

[39] V.N. Şenoğuz and Q. Shafi, Phys. Rev. D 71, 043514 (2005); arXiv:hep-ph/0512170, M. Ur Rehman, Q. Shafi, and J.R. Wickman, Phys. Lett. B 683, 191 (2010).

[40] M. Dine, L. Randall, and S. Thomas, Phys. Rev. Lett. 75, 398 (1995); M.K. Gaillard, H. Murayama, and K.A. Olive, Phys. Lett. B 355, 71 (1995).

[41] D.H. Lyth and T. Moroi, J. High Energy Phys. 05, 004 (2004).

[42] S. Coleman and E. Weinberg, Phys. Rev. D 7, 1888 (1973).

[43] G. Ballesteros, J.A. Casas, and J.R. Espinosa, J. Cosmol. Astropart. Phys. 03, 001 (2006).

[44] K.I. Izawa, M. Kawasaki, and T. Yanagida, Phys. Lett. B 411, 249 (1997); M. Kawasaki, N. Sugiyama, and T. Yanagida, Phys. Rev. D 57, 6050 (1998).

[45] M. Kawasaki and T. Yanagida, Phys. Rev. D 59, 043512
(1999); T. Kanazawa, M. Kawasaki, N. Sugiyama, and T. Yanagida, ibid. 61, 023517 (1999).

[46] M. Kawasaki, M. Yamaguchi, and J. Yokoyama, Phys. Rev. D 68, 023508 (2003); M. Yamaguchi and J. Yokoyama, ibid. 68, 123520 (2003); ibid. 70, 023513 (2004); M. Kawasaki, T. Takayama, M. Yamaguchi, and J. Yokoyama, ibid. 74, 043525 (2006).

[47] C. Pallis, Nucl. Phys. B751, 129 (2006).

[48] G. Lazarides and N. Tetradis, Phys. Rev. D 58, 123502 (1998).

[49] M. Axenides and K. Dimopoulos, J. Cosmol. Astropart. Phys. 07, 010 (2004).

[50] I.G. Moss and C. Xiong, arXiv:hep-ph/0603266 A. Berera, I.G. Moss, and R.O. Ramos, Rept. Prog. Phys. 72, 026901 (2009).

[51] M. Bolz, A. Brandenburg, and W. Buchmüller, Nucl. Phys. B606, 518 (2001); ibid. B790, 336 (2008) (E); J. Pradler and F.D. Steffen, Phys. Rev. D 75, 023509 (2007). 